\title{
Poster session - Abstract 1
}

Centre for inherited metabolic diseases of University Children's Hospital Bratislava about the past and the present

Behúlová D. ${ }^{l}$, Holešová D. ${ }^{l}$, Šalingová A. ${ }^{l}$, Škodová J. ${ }^{l}$, Šebová C. ${ }^{l}$, Perečková J. ${ }^{l}$, Ostrožlíková M. ${ }^{l}$,

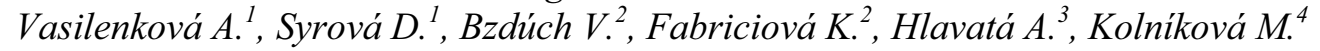

${ }^{1}$ Centre for Inherited Metabolic Diseases, Dept. of Laboratory Medicine, University Children's Hospital, Bratislava, Slovakia

${ }^{2} 1^{\text {st }}$ Dept. of Pediatrics, University Children's Hospital, Bratislava, Slovakia

${ }^{3} 2^{\text {nd }}$ Dept. of Pediatrics, University Children's Hospital, Bratislava, Slovakia

${ }^{4}$ Dept. of Pediatric Neurology, University Children's Hospital, Bratislava, Slovakia

The aim of the report is to present history and achieved results of diagnostic Centre for Inherited Metabolic Diseases. Since 1993, when the former common state was divided, the Centre has been built gradually as a part of the largest Slovak pediatric hospital. At present, the Centre provides biochemical genetic testing including selective screening for inherited metabolic diseases (IMD) as well as lifelong biochemical monitoring of patients with confirmed IMD. Routine and urgent biochemical diagnostics is available in the Centre on the level of wide scale of metabolites in body fluids: ammonia, lactate, 3-hydoxybutyrate, amino acids, organic acids, orotic acid, biotinidase, carnitine, saccharides, oligosaccharides, sialyloligosaccharides, glycosaminoglycans and others. The qualified staff ensures also consultations and expertises for the whole Slovakia. The number of patients increased enormously from 111 to 2936 individuals investigated in 1993 and 2011, respectively. Over the period of the last 19 years, 327 cases with more than 80 various IMD were detected and/or monitored. The most frequent diseases registered were lysosomal disorders, aminoacidopathies, organic acidurias, disorders of carbohydrates and fatty acid oxidation. 


\section{Poster session - Abstract 2}

\section{Organic acidurias in Slovakia}

Škodová $J .{ }^{1}$, Perečková $J .{ }^{1}$, Behúlová $D .{ }^{1}$, Gregová E. ${ }^{2}$, Chandoga $J .{ }^{3}$

${ }^{1}$ Centre for Inherited Metabolic Diseases, University Children's Hospital, Bratislava, Slovakia

${ }^{2}$ Dept. of Clinical Biochemistry, Faculty Hospital of F. D. Roosevelt, Banská Bystrica, Slovakia

${ }^{3}$ Centre of Medical Genetics, University Hospital, Bratislava, Slovakia

Background: The aim of our study was to evaluate organic acidurias (OAU) as rare inherited metabolic diseases (IMD) which has been detected in Slovakia so far. Selective screening for IMD including OAU has been providing in three diagnostic centres of biochemical genetics.

Methods: Profiling of urinary organic acids (OA) has been performed by gas chromatography-mass spectrometry (GC-MS) in two centers in Bratislava and by GC in one department in Banska Bystrica. The investigation of OA was performed in random (morning) urine from children with clinical suspicion of IMD.

Results: Over the period of 18 years (1993-2010), 42 cases of OA metabolism disorders were identified: Canavan disease (12), methylmalonic aciduria (10), propionic aciduria (7), glutaric aciduria type I (2), mevalonic aciduria (2), 3-hydroxy-3-methylglutaric aciduria (2), 2oxoadipic aciduria (2), pyroglutamic aciduria (2), leucinosis (1), multiple carboxylase deficiency (1), 3-methylglutaconic aciduria (1).

Conclusions: It is supposed that many cases of OAU has been undiagnosed in our country. The main cause could be the low number of patients presenting to clinicians suggestive to suspection of inherited metabolic diseases. Insufficient laboratory equipment and lack of qualified staff in biochemical genetic laboratories might contributed to the unsatisfactory results of selective screening of OAU as well. 


\section{Poster session - Abstract 4}

\section{Pompe disease - Diagnostics in Slovakia}

Mattošová S. ${ }^{1}$, Hlavatá A. ${ }^{2}$ Špalek $P .{ }^{3}$, Maceková $D .{ }^{1}$, Chandoga $J .{ }^{1}$

${ }^{1}$ Institute of Medical Biology, Genetics and Clinical Genetics, Comenius University Medical School, University Hospital Bratislava Slovakia.

$22^{\text {nd }}$ Dept. of Pediatrics, Comenius University Medical School, University Children's Hospital Bratislava, Slovakia

${ }^{3}$ Centre for Neuromuscular Diseases, University Hospital Bratislava, Slovakia

Pompe disease (glycogen storage disease type II) is autosomal recessive disorder caused by deficiency of the lysosomal enzyme $\alpha$-glucosidase (acid maltase). This leads to an accumulation of glycogen in various tissues of the body. Pompe disease is classified into infantile, juvenile and adult types based on the clinical onset and presentation of the disorder. Classic infantile form is the most severe form. Infants develop symptoms within the first months of life, presenting with prominent cardiomyopathy, hypotonia and death before 12 month of life due to cardiorespiratory failure. The late-onset forms, classicaly referred to as juvenile and adult onset, have symptoms that are generally limited to skeletal muscle, with a slowly progressive proximal myopathy and marked clinical involvement of respiratory muscles. The structural gene encoding acid $\alpha$-glucosidase (GAA) is located on chromosome 17q25.2-q25.3 and contains 20 exons. To date, more than 289 different variations are listed in the Pompe disease mutation database (www.pompecenter.nl). Of these, 197 have been demonstrated to be pathogenic. The most frequent mutation in Caucasian lateonset Pompe disease patients is leakly IVS1 $(-13 \mathrm{~T} \rightarrow \mathrm{G})$, which gives rise to alternatively spliced transkripts, but still allows for the production of a low amount of normally processed mRNA. This mutation occur in $70 \%$ of patients with late-onset Pompe disease.

We present diagnostic scheme for Pompe disease in our institute. The presence of disease was tested by selective screening by measuring $\alpha$-glucosidase activity in dried blood spots. The diagnosis was confirmed by measuring $\alpha$-glucosidase activity in mixed leukocytes using acarbose as inhibitor of maltase-glucoamylase activity. Based on high prevalence of IVS1 ($13 \mathrm{~T} \rightarrow \mathrm{G}$ ) mutation in late-onset Pompe disease patients we perform detection of this mutation using PCR-RFLP method. We have found this mutation in homozygous form in one patient and in second patient we have found this mutation in heterozygous form. Presence of this frequent mutation we confirmed by sequencing. Sequencing of entire GAA gene will be offered in year 2012 at our institute. 


\title{
Poster session - Abstract 5
}

\section{X-linked Adrenoleukodystrophy in Slovakia}

\author{
Petrovič R., Fischerová M., Konkol’ová J., Chandoga J., Böhmer D.
}

Institute of Medical Biology, Genetics and Clinical Genetics, Medical Faculty, Comenius University, University Hospital, Bratislava, Slovakia

The peroxisome is a single-membrane organelle presents in nearly all eukaryotic cells. Defects in one or more peroxisomal metabolic functions are associated usually with severe clinical manifestations. The group of peroxisomal disorders now includes 17 different diseases.

X-linked adrenoleukodystrophy (X-ALD, MIM\# 300100) is the commonest inherited peroxisomal disorder with a minimum incidence about 1:15000 males. This severe neurodegenerative disorder is characterised by the accumulation of saturated very long chain fatty acids (VLCFA) in the central nervous system, peripheral nerves, adrenal glands and body fluids. The VLCFA accumulation is associated with an impaired capacity for their degradation via $\beta$-oxidation pathway in the peroxisomes and leads to a progressive demyelination of the central and peripheral nervous system and to adrenal cortex insufficiency. The clinical presentation of X-ALD varies widely; at least six phenotypic variants can be distinguished. The two most frequent phenotypes are childhood cerebral form (ccALD) and adrenomyeloneuropathy (AMN).

The X-ALD gene (ABCD1 - GeneID: 215, MIM\# 300371) localised in Xq28 encodes a peroxisomal membrane protein that belongs to ATP-binding cassette (ABC) superfamily of transporters. ABCD 1 comprises 10 exons spanning approximately $21 \mathrm{~kb}$ of genomic DNA, encodes an mRNA of 4.3 kilobases and a protein of 745 amino acid residues. More than 580 different mutations have been reported in gene (www.x-ald.nl) but no correlation between genotype and phenotype has been found. Diagnosis of peroxisomal disease is based on morphological, biochemical, immunochemical and molecular genetic examinations.

The laboratory diagnostic of peroxisomal disorders in Slovakia is performed exclusively in our Institute has been started in 1996. During this period we have diagnosed more than 20 patients with X-ALD in 14 unrelated families. This fact facilitates genetic counselling activity and enables prenatal diagnosis in these families. In addition, we also increased the mutational spectrum of ABCD1 gene by describing of 4 novel mutations in this gene.

Acknowledgment:This work was supported by Ministry of Health of the Slovak Republic under the project "Inherited peroxisomal disorders - development and translation of laboratory, clinical, diagnostic and therapeutic algorithms to practice" no. 2007/38 -FNSPBA-03. 


\section{Poster session - Abstract 6}

\section{Homogentisate 1,2-dioxygenase (HGD) gene variants causing alkaptonuria (AKU) in the World and in Slovakia}

Kádaši L. ${ }^{1,2}$, Radvanský J. ${ }^{1}$, Gallagher J. A. ${ }^{3}$, Jarvis $J . C^{3}{ }^{3}$, Ranganath L. ${ }^{3}$, Usher ${ }^{3}{ }^{3}$, Alsbou M. S. , Zat'ková A. ${ }^{1,2}$

${ }^{1}$ Institute of Molecular Physiology and Genetics, Slovak Academy of Sciences, Bratislava, Slovakia

${ }^{2}$ Dept. of Molecular Biology, Faculty of Natural Sciences, Comenius University, Bratislava, Slovakia

${ }^{3}$ Dept. of Musculoskeletal Science, Institute of Ageing and Chronic Disease, University of Liverpool, United Kingdom

${ }^{4}$ Dept. of Pharmacology, Faculty of Medicine, Mutah University, Karak, Jordan

Alkaptonuria (AKU) is rare autosomal recessive disorder caused by mutations in the homogentisate 1,2 dioxygenase (HGD) gene leading to a deficiency of HGD activity. AKU is characterized by homogentisic aciduria, deposition of ochronotic pigment in connective tissues and painful and disabling arthritis.

In order to curate and record AKU causing mutations and also benign HGD variants, we have created an HGD mutation database that is available online (http://hgddatabase.cvtisr.sk/). At present the database contains 152 unique HGD variants (129 public), out of which 118 have been reported as pathogenic mutations in about 270 AKU families. We also present an interesting geographical distribution of HGD allelic variants, including novel cases and mutations from Slovakia, South Korea, Great Britain and Jordan. AKU shows a very low prevalence in most ethnic groups $(1: 250,000)$, but in Slovakia and the Dominican Republic the incidence raises up to 1:19000. About 220 patients have been reported in Slovakia, a country with population of approximately 5 millions. Most of AKU cases come from a small mountainous region in the north-west part of Central Slovakia (population of about $125,000)$ that was geographically isolated until late 1950s. However, we identified 12 different HGD mutations in this region, which excludes a founder effect as the simple explanation of this remarkable distribution. Employing haplotype analysis, we compared AKU chromosomes carrying identical HGD mutation in the patients originating from different countries. We observed that 7 out of 12 mutations were most likely to have originated in Slovakia. Moreover, these allelic variants occur in previously described mutational hot-spots within the HGD gene ("CCC" triplets, $\mathrm{CpG}, \mathrm{c.} .342+1 \mathrm{G}$ ), indicating an increased mutation frequency within the gene in this particular region of Slovakia, and therefore providing an explanation for the higher incidence of AKU in the country. However, it remains unclear which mechanism could have acted specifically on the HGD gene to increase its mutation rate. 


\section{Poster session - Abstract 7}

\section{Molecular diagnostics of myotonic dystrophy type 1 and type 2 by multiplex PCR}

Radvanský $J^{1,2}$, Ficek $A^{1,2}$, Kádaši L., ${ }^{1,2}$

${ }^{1}$ Institute of Molecular Physiology and Genetics, Slovak Academy of Sciences, Bratislava, Slovakia

${ }^{2}$ Dept. of Molecular Biology, Faculty of Natural Sciences, Comenius University, Bratislava, Slovakia

Myotonic dystrophy type 1 (DM1) is caused by expansion of a (CTG)n repeat in the DMPK gene, while myotonic dystrophy type 2 (DM2) is caused by expansion of a (CCTG)n part of a complex repetitive motif (TG)n(TCTG)n(CCTG)n. The size of healthy range alleles is highly variable in both loci and expanded alleles may contain several thousands of repeats in both DM1 and DM2. Combination of conventional PCR and "triplet" or "tetraplet" repeatprimed PCR (TP-PCR) is a commonly used approach to characterize healthy range alleles and to prove the presence or absence of expanded alleles in molecular testing for both disorders. We have designed three multiplex PCR reactions for simultaneous characterisation of the DM1 and DM2 locus using PCR based analyses and capillary electrophoresis on automatic DNA analyser. The first reaction includes bi-directionally labelled conventional PCRs for both the DM1 and DM2 locus. The second reaction contains the reverse directional DM1 TP-PCR and the reverse directional DM2 TP-PCR, while the third reaction was designed to include the forward directional DM1 TP-PCR and the forward directional DM2 TP-PCR. These three reactions were designed to be performable under the same amplification and electrophoretic conditions allowing thus their parallelisation. The usefulness of our multiplex assay is underlined by suggestions from previous studies, which showed, that performing conventional PCR with bi-directional labelling of specific amplicons and TPPCRs simultaneously from both sides of the repeat regions can increase the reliability and accuracy of the assays which are based on the described methods. 


\section{Poster session - Abstract 8}

Acquired neuromyotonia - a rare autoimmune disease of neuromuscular transmission

Špalek $P .{ }^{1}$, Martinka I. ${ }^{1}$, Vincent $A .^{2}$

${ }^{1}$ Centre for Neuromuscular Diseases, University Hospital Bratislava, Slovakia

${ }^{2}$ Institute of Molecular Medicine, Neuroscience Group, John Radcliffe Hospital, Oxford, United Kingdom

Background: Neuromyotonia (NMT) is a rare disorder of spontaneous and continuous muscle fibre activity of peripheral nerve origin. There are two main forms of NMT, inherited and acquired. Most acquired cases of NMT have autoimmune origin with autoantibodies against voltage-gated potassium channels (VGKC) that are present on the presynaptic motor nerve terminals (,autoimmune channelopathy"). Loss of these VGKCs leads to prolonged depolarisation, prolonged opening of calcium channels and excess acetylcholine release.

Case report: We report 30-year-old male patient with 4-year-history of myokymia, muscle stiffness, muscle cramps, delayed relaxation after contraction (pseudomyotonia) and hyperhidrosis, brought on by exercise, later the complaints were continuous. A spontaneous burst of single motor-unit activity and neuromyotonic discharges 100 to $170 \mathrm{~Hz}$ were the most characteristic features found in EMG studies. The diagnosis of acquired neuromyotonia was confirmed by evidence of serum antibodies against VGKCc (266 pmol/l). Plasma exchange and combined long-term immunosupressive therapy (prednisone and cyclophosphamide) resulted in marked and sustained improvement.

Conclusion: Acquired autoimmune NMT is a progressive disabling disorder, therefore early diagnosis and early imunotherapy is of significant importance for the prognosis of NMT. 


\section{Poster session - Abstract 9}

\section{Clinical and radiographic pictures of ochronotic arthropathy}

Rovenský J., Krátka M.

National Institute of Rheumatic Diseases, Piešt'any, Slovakia

Alkaptonuria is an inherited disorder of metabolism of aromatic aminoacids phenylalanine and tyrosine. The defect activity of the enzyme homogentisic acid oxidase leads to the accumulation of homogentisic acid in organism and its excretion in urine. Its polymer the ochronotic pigment impregnates the bradytrophic tissues. In clinical and laboratory pictures, pigmentation of scleras and ears can be seen, which are functionally insignificant; restricting changes of the locomotory system, as well as presence of homogentisic acid in urine can be found. The patients are generally of lower height and they indicate further decrease of their height. The average height of our patients with ochronotic arthropathy is $158.5 \mathrm{~cm}$ in men and $151 \mathrm{~cm}$ in women. Compared to the overall population, men are lower by $12.9 \mathrm{~cm}$ and women by $6.1 \mathrm{~cm}$. Their height even decreases with the progress of the disease.

First subjective as well as objective symptoms are localized in the area of vertebral column. The patients indicate an undefined feeling of stiffness in the lumbosacral area, which might be accompanied by a minor pain. Some patients feel a certain block while back straightening, which can be overcome only with pain. This stiffness is later accompanied by a significant pain, which is however less severe than in inflammatory diseases of the spine. The pain remains localized and limited to the lumbal area without any radiation into surrounding areas and without a periodical fluctuation in its strength. It seems, that the disproportion between the less significant pain and the progressive movement restriction is typical for ochronotic arthropathy.

Among radiographic changes in manifest ochronosis, we repeatedly found a formation of marginal osteophytes of the vertebral bodies, which were often very massive. Almost always, smaller porotic preudocystic deposits were found in the imminence of those osteophytes. Regressive changes of the discs accrue and calcifications become more intense with further development of the diesase process. 


\section{Poster session - Abstract 10}

\section{Orphan disease in hemostasis: Results from the National Registry of thrombophilic states in Slovakia}

Plameňová I., Sokol J., Dobrotová M., Ivanková J., Chudý P., Staško J., Kubisz P.

National Center of Hemostasis and Thrombosis, Jessenius Faculty of Medicine, Comenius University, Martin, Slovakia

A rare disease, also referred to as the orphan disease, is any disease that affects a small percentage of the population (fewer than 1 in 2000 people, according to the European Commission on Public Health).

Thrombophilia is an inherited or acquired disorder of hemostasis that leads to the increased tendency of thrombosis and predisposes patients to thrombosis and to its recurrence. Clinically hereditary and acquired trombophilias participate in onset of venous thromboembolism and arterial thrombosis or recurrent spontaneous fetal loss. According to the Conception of health care in Hematology and transfusiology (Bulletin of MH SR, issue of 31.8.2006; vol. 54, p. 13-18) the National Centre of Hemostasis and Thrombosis seated at Department of Hematology and Transfusiology, Jessenius Medical Faculty of Commenius University and University Hospital in Martin preserves the health care predominantly in patients with inherited and acquired trombophilias in the whole Slovak republic and manages the National Registry of Thrombophilic States. The aim of the National registry of thrombophilic states is to create a functional and flexible database with the appropriate software and practical applications, especially for those who take care about thrombophilic patients. On 25th January 2012, 5171 patients are registered here. The majority of these cases are not orphan diseases. We present only results concerning orphan thrombophilic states according to the register of orphan diseases (orpha.net).

a) Congenital antithrombin III (AT III) deficiency, ORPHA82, ( $\mathrm{n}=165,3 \%)$

b) Congenital Hageman factor deficiency (FXII), ORPHA330, $(n=119,2,3 \%)$

c) Congenital protein $\mathrm{C}$ deficiency, ORPHA745, $(\mathrm{n}=133,2,6 \%)$

d) Congenital ORPHA743 and acquired ORPHA26349 protein $\mathrm{S}$ defic., $(\mathrm{n}=489,9,45 \%)$

e) Familial dysfibrinogenemia ORPHA98881, $(\mathrm{n}=84,1,6 \%)$

f) Hypoplasminogenemia (plasminogen deficiency type I) ORPHA722, ( $\mathrm{n}=6,0,1 \%$ )

g) Familial thrombodulin anomalies ORPHA3324, $(\mathrm{n}=1,0,02 \%)$

h) Antiphospholipid syndrome ORPHA80, ( $\mathrm{n}=248,4,7 \%)$

i) Heparin-induced thrombocytopenia ORPHA3325, $(\mathrm{n}=3,0,05 \%$, only patients from the University Hospital in Martin)

j) Thrombotic thrombocytopenic purpura ORPHA54057, $(\mathrm{n}=17,0,3 \%$ - only patients from the middle Slovak region)

We would like to mention orpha.net classification is still not complete and should be reassessed. We suppose to include acquired APC resistance (phenotype), hyperhomocysteinemia, sticky platelet syndrome and others.

Acknowledgement: Supported by the European Regional Development Fund (ERDF) Project CEPV II (Center of Excellence for Perinatological Research) (ITMS 26220120036), Project CEVYPET (ITMS 26220120053) and grant Vega 1/0029/11.

Acta Facult. Pharm. Univ. Comenianae, 2012, 59, Suppl 1 


\section{Poster session - Abstract 11}

\section{Monogenic diabetes: The Slovak experience}

Klimeš I. ${ }^{1}$, Staník J. ${ }^{2,}{ }^{1}$, Hučková M. ${ }^{1}$, Valentínová L. ${ }^{1}$, Masindová $I .{ }^{1}$, Barák L. ${ }^{2}$, Pašková M. ${ }^{3}$, Monogenic Diabetes Study Group and Gašperíková D. ${ }^{l}$

${ }^{1}$ DIABGENE Laboratory, Institute of Experimental Endocrinology, Slovak Academy of Science, Bratislava, Slovakia

${ }^{2} 1$ st Dept. of Pediatrics, Children's Diabetes Center, Comenius University, Bratislava, Slovakia

${ }^{3}$ Children's University Hospital Košice, Slovakia

Monogenic defects of insulin secretion belong to the few chapters of medicine where the accessibility of DNA diagnostics in Slovakia started in 2004 when the DIABGENE laboratory was founded. The aim of this presentation has been to provide you with 1) the epidemiology of monogenic diabetes /M-DM/ in Slovakia, 2) with the MODY patients mutation profile, 3) brief information on relevant structure-function studies, and with 4) the DNA diagnostic-based changes of therapy with sulphonylurea (SU) of permanent neonatal diabetes (PND) and in HNF1A MODY diabetics. We have been recruiting M-DM patients since 2004 all over Slovakia. DNA sequencing has been carried out for relevant 9 genes. Ad 1) We received blood from 729 patients of 252 families with a clinical suspicion for MDM. DNA sequencing confirmed a molecular disease cause in 160 patients of 64 families. This corresponds to $22 \%$ of the patients analyzed. The prevalence of MODY-DM in Slovakia is thus 30 pts per million of inhabitants. The most prevalent "MODY" subtype was the GCKdiabetes (46 probands and 77 family relatives), followed tightly by HNF1A diabetes (25 probands and 35 family relatives). Our numbers are very close to the Czech data. Ad 2) Analysing data for hsCRP (as measured with the Oxford technology) and their association with the HNF1A in our patient cohort has clearly proven validity of the Owen's concept that low hsCRP levels could become a useful biomarker of M-DM in the clinical practice. That might increase the capture rates of monogenic DM subjects. Ad 3) Most of the "MODY" mutations were not novel or unpublished, except of the 8 GCK mutations, 4 HNF1A and 1 HNF4A mutation; all co-segregated with glycemia. Ad 4) Molecular pathogenicity of new GCK mutations was investigated and elucidated. In addition, a set of intensively sequenced MODY-X patients with negative outcome of DNA diagnostics with a "typical" MODY HNF1A phenotype were sent to Oxford for NextGen sequencing. Ad 5) With help of the Slovak Diabetes Registry, also all patients with PND were identified, i.e. 9 cases of PND borne since 1981. This has generated new and generally accepted prevalence of this relatively rare disease of 1 to 200000 live borne kids. We have also patients who underwent the therapy change from insulin to SU. Long- term ( 5 yrs) follow-up of the PND pts has clearly shown the usefulness of the SU treatment. SU is a good option to switch from insulin treatment also in the HNF1A subjects. DIABGENE, the Slovak only DNA diagnostic centre for M-DM, is engaged into intensive research collaboration. Due to its broad clinical background and ongoing development of new methodologies for different monogenic diseases it is best suited for translation of the research findings into clinical practice.

Acknowledgment:This work was supported in part by research grants ERDF (Transendogen/26240220051), APVV-51-014205, VEGA 2/0151/11 and Slovak Diabetes Association

Acta Facult. Pharm. Univ. Comenianae, 2012, 59, Suppl 1 


\section{Poster session - Abstract 12}

\section{Parkinson disease treatment by continuous levodopa therapy}

Kračunová K., Cibulčík F., Benetin J.

Neurology Clinic of Slovak Medical University and University Hospital in Bratislava, Slovakia

Parkinson disease (PD) affects motor system and is clinically manifested by characteristic motor dysfunctions consisting in four major symptoms - tremor, bradykinesia, rigidity and postural instability. Advanced PD is characterized by gradual failure of dopamine replacement therapy and manifested as motor and non-motor fluctuations and worsened patient's autonomy. Patients suffer from autonomic dysfunction, neuropsychiatric disorders, sleep problems and other difficulties not present in PD early stages.

The levodopa/carbidopa intestinal gel therapy shows improvement of motor functions and increased quality of life without a rise of dyskinesia occurrence. The therapy is beneficial for patients with advanced PD who are still able to respond to levodopa therapy when a peroral therapy does not bring sufficient results. Levodopa/carbidopa gel is administered by a portable pump dosing the drug directly into the small intestine where the active substance is being absorbed directly into blood. In opposite to standard medication, gel is not accumulated in the stomach and its administration into small intestine enables immediate and continuous absorption.

In the EU, 2.4 persons out of 10.000 or, 90.000 in total, suffer currently from advanced PD. In Slovakia, levodopa/carbidopa gel treatment has been available since 2009. Nowadays, the therapy is administered to 14 patients. According to epidemiologic data, it should be appropriate to almost 300 Slovak patients. Our experience shows that it means substantial improvement of mobility, reduction of dyskinesia and overall improvement of the quality of life. 


\section{Poster session - Abstract 13}

\section{Plerixafor - orphan drug in different stem cells mobilization approaches}

Zwiewka M., Štefániková Z., Masárová K., Martinka J., Hrubiško M., Pribulová H., Hapalová J., Bojtárová E., Mistrik M.

Dept. of Hematology and Transfusiology, University Hospital Bratislava, Slovakia

Introduction: Autologous peripheral blood stem cell transplantation is essential for long-term survival and quality of life in multiple myeloma patients. For successful transplantation a mobilization of stem cells in bone marrow with subsequent collection from peripheral blood is needed. In the pre-plerixafor era poor mobilizers were a problem uneasy to solve. Now different strategies of plerixafor use are available and reduce the percentage of poor mobilizers to $2 \%$.

Methods: In our center we used two different approaches for plerixafor treatment. First was standard remobilization using G-CSF + plerixafor scheme $(\mathrm{G}+\mathrm{P})$. Second approach was on demand use in a failed first-line mobilization attempt $(\mathrm{C}+\mathrm{G}+\mathrm{P})$.

Results: Both mobilization strategies allowed to collect enough CD34+. Duration of cytopenia after transplantation was comparable to patients mobilized with $\mathrm{C}+\mathrm{G}$ only. Significant reduction of toxic granulation in patients mobilized with plerixafor may be a proof of lesser number of infectious complications. We also proved that chemomobilization with cyclophosphamide decreases regenerative potential of bone marrow.

Conclusions: Avoiding chemomobilization in future mobilization regimes could prevent possible neutropenia and its complications. However due to our FACS analysis we believe that most valuable potential of plerixafor may be in curative regimens of diseases which have affinity to bone marrow. 


\section{Poster session - Abstract 14}

\section{Paroxysmal nocturnal hemoglobinuria treatment with hematopoetic stem cell transplantation}

Bojtárová E., Žiaková B., Kubalová S., Mistrík M.

Dept. of Hematology and Transfusiology, University Hospital Bratislava, Slovakia

Aim: Paroxysmal nocturnal hemoglobinuria (PNH) is a rare clonal hematopoetic stem cell disease which can lead to life-threatening complications (intravascular hemolysis, thrombotic events and kidney disease).

Patients and methods: We present data from 6 patients (pts) with PNH who underwent hematopoetic stem cell transplantation (HSCT) in 2001-2011: 4 from match HLA-identical sibling and 2 from matched unrelated donor (MUD). Indication for allogeneic HSCT was aplastic/hypoplastic bone marrow (2/6 pts), severe course of PNH with hemolytic crisis and/or thrombosis (5/6 pts), transfusion dependency (6/6 pts). Conditioning regimen included total body irradiation (TBI) and cyclophosphamide (CY $60 \mathrm{mg} / \mathrm{kg} 2$ days) and antithymocyte globulin (ATG) in $3 \mathrm{pts}$, fludarabine (FLU $30 \mathrm{mg} / \mathrm{m} 2$ for 4 days) and busulfan (BU $4 \mathrm{mg} / \mathrm{kg} 2$ days) in 1 pt. Before MUD FLU 30mg/m2, BU $4 \mathrm{mg} / \mathrm{kg} 2$ days and ATG (2 pts). Graft versus host disease (GvHD) prophylaxy was provided by methotrexate (MTX) + cyclosporin A in 5 patients, in 1 patient MTX was replaced with mycophenolate mofetil because of serious gastrointestinal complications. Source of stem cells were mainly peripheral blood stem cells (5 patients) with median $7.4 \times 10^{\wedge} 8$ nuclear cells $/ \mathrm{kg}(1.6-12.3)$ and $5.2 \times 10^{\wedge} 6 \mathrm{CD} 34+$ cells $/ \mathrm{kg}$ (3.6-5.8).

Results: Engraftment was observed in all patients: median of neutrophil count above $0.5 \mathrm{G} / 1$, above $1.0 \mathrm{G} / 1$ and trombocyte count above $50 \mathrm{G} / 1$ were achieved 23(13-23), 23.5 (14-26) and 23.5(13-132) days after HSCT. Acute GvHD (intestinal form gr.II-III) developed in 1 patient, 1 has mucosal and ocular signs of chronic GvHD. To date 30.11.2011, 36.5(0.7-121) months after HSCT all patients are alive and don't require treatment of PNH.

Conclusion: Allogeneic HSCT provides effective and safe curable treatment for PNH. 


\section{Poster session - Abstract 15}

\section{Experience with Treatment of Mucopolysaccharidoses in Slovakia}

Hlavatá A., ${ }^{1}$ Kubina M., ${ }^{1}$ Šaligová J., ${ }^{2}$ Poupětová H., ${ }^{3}$ Šalingová A., ${ }^{4}$ Chandoga J.

${ }^{1} 2^{\text {nd }}$ Dept. of Pediatrics, Comenius University Medical School, University Children's Hospital, Bratislava, Slovakia

${ }^{2}$ Children's Hospital, Šafárik University, Košice, Slovakia

${ }^{3}$ Institute of Inherited Metabolic Disorders, First Faculty of Medicine, Charles University in Prague and General University Hospital in Prague, Czech Republic

${ }^{4}$ Dept. of Laboratory Medicine, Children's Hospital, Bratislava, Slovakia

${ }^{5}$ Centre of Medical Genetics, University Hospital, Bratislava, Slovakia

Background: Mucopolysaccharidoses (MPS) are a set of rare lysosomal storage diseases. The absence of a intralysosomal degrading enzyme leads to a continuous accumulation of biopolymers in various organ tissues. It has been recently made possible to treat some of the eight known subtypes of MPS with enzyme replacement therapy (ERT).

Patients and methods: We report on the treatment and management of six patients with MPS (one patient with MPS VI, one patient with MPS II and four patients with MPS I).

Results: Our first patient with MPS I initiated the treatment at the age of 10 years. There was a significant decrease in the frequency of the respiratory tract infections, however the somatic deformities did progress which in turn limits the mental development.

Two sibling patients with Morbus Scheie commenced the treatment at the age of 6 and 12 years. The receding of the contractures is apparent predominantly in the younger patient. The last patient with MPS I is a 2 year old girl who was started on ERT at the age of 15 months. There has been improvement as regards to organomegaly and patient's mental development, however dysmorphic features are still prominent. Patient with MPS VI treated from the age of 3 years responded to the treatment considerably. MPS II patient initiated the treatment at the age of 2.5 years. There was a significant improvement in the biochemical markers, however with only slow regression of the somatic features.

Conclusion: The experience with ERT in our clinical setting are in accordance with previously published data, that is, the effect of ERT depends on the age of the patient at therapy initiation. Patient's family plays major role in the outcome and mitigation of the rate of progression of the disease. The long term effect and efficacy of ERT is to be determined in the years to come. 


\section{Poster session - Abstract 16}

\section{Long-term secondary prophylaxis with recombinant activated factor VII (rFVIIA) in haemophilia A with inhibitor - case report}

\section{Chudý P., Plameňová I., Dobrotová M., Ivanková J., Sokol J., Staško J., Kubisz P.}

National Center of Hemostasis and Thrombosis, Jessenius Faculty of Medicine, Comenius University, Martin, Slovakia

We describe a patient with haemophilia A (ORPHA 169 802, ICD code: D66, MIM number: 306 700), FVIII inhibitor titer and haemophilic arthropathy in whom long-term secondary prophylaxis with recombinant activated factor VII (rFVIIa; NovoSeven ${ }^{\circledR}$ ) was initiated following unsuccessful immune tolerance induction (ITI). The patient, born in 1995, was diagnosed with moderately severe haemophilia $\mathrm{A}$ at the age of three years following repeated mucosal and joint bleeds since the first year of life. On-demand treatment with human FVIII over two years (50 exposure days) resulted in generation of FVIII inhibitor $(38.2 \mathrm{BU} / \mathrm{ml}$, 09/2000). ITI was administrated (09-12/2001) using a high-dose regimen (Bonn protocol), because of inhibitor titer $10.8 \mathrm{BU} / \mathrm{ml}$ and repeated frequent bleeds. But ITI was not finished due to patients's desire to avoid repeated catheter placements and venipunctures. The patient continued to receive bypassing agents on demand; however, at his parent's decision, he did not receive any treatment between 01/2007-12/2007 and was not regularly examined. In December 2007, the patient was admitted to hospital. He required assistance to walk and showed the limited mobility of the left knee (extension deficiency of $40^{\circ}$ ) and intra-articular haemorrhage. Laboratory tests indicated severe haemophilia with high-titre inhibitor $(8,5$ $\mathrm{BU} / \mathrm{ml}$ ). Prophylactic rFVIIa regimen (rFVIIa $90 \mathrm{ug} / \mathrm{kg}$ three times daily) was initiated and patient underwent intensive physiotherapy with prophylactic FVIIa coverage $(120 \mathrm{ug} / \mathrm{kg}$ three times a week). After four months (04/2008) patient was able to walk unassisted for longer distances. Hypotrophy and oedema of the left knee persisted, but flexion was almost normal, with slightly improved extension despite a persistent deficiency of $25^{\circ}$. Rehabilitation and reduced doses of rFVIIa continued at home. The inhibitor titer of FVIII continuosly decreased $(0.6 \mathrm{BU} / \mathrm{ml})$. At this time the second ITI is ongoing.

Acknowledgement: Supported by the European Regional Development Fund (ERDF) Project CEPV II (Center of Excellence for Perinatological Research) (ITMS26220120036), Project "Support of human resources development using the most modern methods and forms of education at JFM CU in Martin"(ITMS 26110230031) and grant Vega 1/0029/11. 


\section{Poster session - Abstract 17}

Our experience with the use of the interleukin-1 receptor antagonist in the treatment of the patient with CINCA/NOMID syndrome (case study - 5 years of therapy)

Vargová ${ }^{l}{ }^{1}$, Macejová, $Z^{2}$

${ }^{1} 1^{\text {st }}$ Dept. of Pediatrics, P. J. Šafárik University and Children Hospital, Ko3ice, Slovakia

${ }^{2} 3^{\text {rd }}$ Dept. of Internal Medicine, P. J. Šafárik University and University Hospital, Košice, Slovakia

Background: CINCA/NOMID is an autoinflammatory disorder characterized by the triad: neonatal onset of cutaneous symptoms, chronic meningitis, and recurrent fever. It belongs to the group of cryopyrinopathies -a rare hereditary autoinflammatory syndrome caused by excessive production of interleukin-1beta.

Objectives: To evaluate effectiveness and safety of the 5 year therapy with interleukin -1 receptor antagonist in patient with CINCA/NOMID syndrome.

Methods: Effectiveness of the therapy was evaluated on the basis of patient's subjective complaints (exathem, headaches, pain in joints, changes in well-being), objective findings and a value of specific markers of inflammation.

Results: During the first week of therapy the patient reported dramatic relief of symptoms she suffered for years. Gradually levels of inflammation markers significantly decreased and bouts of fever dissapered. At present the patient is still closely monitored, she continues to be free of any significant subjective clinical symptoms. Nevertherless, the loss of vision and impairment of hearing slowly progressing.

Conclusions: Long -term therapy with anakinra in patient with CINCA/NOMID resulted in permanent relief of common clinical symptoms as well as decrease in levels of inflammation indicators .However, the treatment could not reverse progression of hearing loss (probably due to delay of starting therapy). 


\section{Poster session - Abstract 18}

\section{A single centre experience with connective tissue associated pulmonary arterial hypertension}

Luknár M. ${ }^{1}$, Lesný P. ${ }^{1}$, Varga I. ${ }^{1}$, Solik P. ${ }^{1}$, Lukáč $J .{ }^{1}$, Rovenský $J .{ }^{1}$, Tuchyňová A. ${ }^{2}$, Goncalvesová E. ${ }^{1}$

${ }^{1}$ Pulmonary Arterial Hypertension Centre, National Cardiovascular Institute, Bratislava, Slovakia

${ }^{2}$ National Institute of Rheumatic Disease, Pieštany, Slovakia

Background: Pulmonary arterial hypertension (PAH) is a rare disease and can be associated with connective tissue diseases (CTD) and strongly precludes a negative prognosis. Pts with CTD associated PAH have been proven to benefit from specific treatment. Right heart catheterization is essential for the correct diagnosis.

Aim: We tried to describe the prevalence of PAH among patients with CTD referred to a PAH centre due to clinical and/or echocardiographic suspicion of PAH.

Patients and methods: Eighty seven patients were presented to PAH centre by rheumatologists due to either clinical or echocardiographic suspicion of PAH from May 2007 to August 2011. All pts had preserved left ventricular ejection fraction and no significant left heart valvular disease. Scleroderma spectrum of disease (mostly limited form) was the underlying CTD in 65 pts, systemic lupus in 6 pts, dermatomyosititis and polymyositis in 3 pts, rheumatoid arthritis in 3 pts, primary Sjogren syndrome in 2, overlap syndrome in 3, and other CTD in 5 pts. PAH suspicion was discarded in 38 pts due to a lack of clinical and echocardiographic signs or for obvious other reasons for $\mathrm{PH}$ such as marked lung disease. Catheterization was considered in 49 pts and finally performed in 38 pts (11 pts refused the invasive procedure). Other classes of $\mathrm{PH}$ were confirmed or ruled out by a comprehensive diagnostic algorithm.

Results: Sixteen patients had no PH according to current criteria. From among 22 pts with pulmonary hypertension, 4 had class II PH due to left ventricular diastolic dysfunction. Class III PH due to hypoxia was confirmed in 3 pts. Eventually, 15 pts were diagnosed with PAH. In 11 pts, the underlying CTD was scleroderma, 2 pts had rheumatoid arthritis, 1 Sjogren syndrome, and 1 patient lupus. These patients were treated by specific PAH treatment.

Conclusion: PAH was present in $17.2 \%$ of CTD pts referred due to clinical and/or echocardiographic suspicion. In the scleroderma subgroup, the prevalence of PAH was $16.9 \%$. This does not reflect the actual prevalence of PAH associated with CTD due to group characteristics and other factors. Catheterization is indicated in symptomatic pts even in the absence of marked echocardiographic signs of $\mathrm{PH}$. 


\section{Poster session - Abstract 19}

\section{Pulmonary arterial hypertension - trends in costs changes}

Foltánová $T{ }^{1}$, Tomáš $S .{ }^{l}$, Petrová L. ${ }^{2}$, Kuželová M. ${ }^{1}$, Kákošová $V .{ }^{3,}$

${ }^{1}$ Dept. of Pharmacology and Toxicology, Faculty of Pharmacy, Comenius University, Bratislava, Slovakia

${ }^{2}$ Dept. of Management and Organisation in Pharmacy Faculty of Pharmacy, Comenius University, Bratislava, Slovakia

${ }^{3}$ Dept. of Pharmacy, University Children's Hospital, Bratislava, Slovakia

Introduction: The European medicine agency (EMA) implemented in the year 2000 specific policies to stimulate the innovation in the fields of rare disease research and orphan medicinal products (OMP) development. However price and reimbursement are in charge of every member state. Although orphan drugs pricing follows the same economic logic as pricing generally, the monopolic power of orphan drugs results in high prices. At the example of pulmonary arterial hypertension, we present the price development of OMP in the Slovak Republic.

Aim: In the SR, the information about direct costs for treatment of rare diseases with OMP were not published yet. Bosentan is the first OMP available on the Slovak market already 6 years (up september 2005). Thus why we bring short overview of direct costs for treatment of PAH with OMP in the SR in the time period 122005-012011.

Methods: For the analysis we used the data from National insurance company, where most of the patients with rare diseases is insured. We studied basic demographic parameters (gender and age), type of OMP as well as total number of packages per year. The costs of pharmacotherapy are presented as total costs for treatment of PAH with OMP during the studied period as well as price changes of every OMP during the period.

Results: Together 100 patients with PAH were treated (M/F 33/67\%, 8-82 yrs). Out of them 11 were children $(\mathrm{M} / \mathrm{F} 18 / 82 \%, 8-17 \mathrm{yrs})$. We noticed an increasing trend in number of patients treated with OMP per year (Table 2). Most frequently used OMP was bosentan (59\%), followed by sildenafil $(33 \%)$ and ambrisentan $(8 \%)$. The total costs for OMP were 3 290981.9 euro In the figures we present the price reduction per package of all OMP. It is presented as price reduction in total as well as price reduction against the previous year. Average price reduction between two years was 12 resp. 14 resp. 9\% resp. 4\% resp. 31\%. (Tracleer 62,5mgx56, Tracleer 125mgx56, Revatio 20mgx60, Volibris10mgx30, Volibris 5mgx30). The highest price reduction was by Revatio 20 mgx90 (69\%).

Conclusion: Although the very high price of OMP and their monopolic power entering the slovak market is reflected in better diagnosing and increased number of treated rare patients. Slovak national health policy implemented processes (as 4 times per year cathegorisation) which help to negotiate the price more frequently thus meeting the societal needs and saving the public health resources.

Literature: Garin MC, Clark L, Chumney EC, Simpson KN, Highland KB. Cost-utility of treatments for pulmonary arterial hypertension: a Markov state-transition decision analysis model. Clin Drug Investig. 2009;29(10):635-46.; Simoens S. Pricing and reimbursement of orphan drugs: the need for more transparency OJRD 2011; 6:42:1-8; ESC/ERS 2009, The Task Force for the Diagnosis and Acknowledgement: This work was supported by FaF UK 36/2011

Acta Facult. Pharm. Univ. Comenianae, 2012, 59, Suppl 1 


\title{
Poster session - Abstract 20
}

\section{Analysis of real access of drugs for treatment of rare diseases}

\author{
Kuželová M., Kosíková L., Ondriašová E., Foltánová T.
}

Dept. of Pharmacology and Toxicology, Faculty of Pharmacy, Comenius University, Bratislava, Slovakia

Introduction: The availibility of orphan drugs (OD) at the market in member states seems to be a big limitation of OD policy. The aim of this analysis was to identify the availability of orphan drugs (OD) in the Slovak Republic. Since in the Slovak Republic the drugs are available for the patients when they get their reimbursement class - are categorized, we analyzed the duration of implementation into the categorization list.

Material and methods: We analyzed data between May 2004, when the Slovak Republic joined EU, and December 2009. We used data from the European Medicines Agency website (list of authorized orphan drugs) and Slovak Ministry of Health website (categorization list). Further used data were ATC codes, date of authorization and date of implementation into the categorization list. Real availability of the OD was calculated as the difference between date of EU authorization and date of categorization in the Slovak Republic.

Results: 59 OD were granted an EU marketing authorization up to 1. 1.2010. The most of the OD were authorized in ATC group L (antineoplastic and immunomodulating agents) - 27 (45.7\%) and group A (alimentary tract and metabolism) - $12(20.3 \%)$. In the same period, in SR, 24 OD (40.7\% all OD) were categorized; most of them were from ATC group L - 9 (37.5\%) and group A - 6 (25\%). All OD in the Slovak Republic are 100\% reimbursed. However only 8 OD met the legislative demand concerning the time from authorization to categorization of the medical product (12 months). The rest (16 OD) reach the Slovak market with average delay $14[1-38]$ months.

Conclusion: Although the Slovak healthcare policy is friendly to OD and all OD have $100 \%$ reimbursement, only $40.7 \%$ of OD reached the market. Our analysis also highlighted that there are still limits in fast OD implementation into the categorization list and thus their real availability.

Literature: Butlen-Ducuing F, Riviere F, Aarum S, Llinares-Garcia J.: European Medicines Agency support mechanisms fostering orphan drug development. Drug News Perspect 23, 1, p. 71-81, 2010; Committee for Orphan Medicinal Products and the European Medicines: European regulation on orphan medicinal products: 10 years of experience and future perspectives. Nat Rev Drug Discov 10, 5, p. 341-349. 2011 


\section{Poster session - Abstract 21}

\section{Organization of neonatal screening in Slovakia}

Knapková M., DluholuckýS.

Newborn Screening Centre Slovak Republic, Children's Faculty Hospital, Banská Bystrica, Slovakia

Background: Population - based neonatal screening is important preventive programe. In Slovakia was established in 1972 with neonatal screening (NS) for phenylketonuria (PKU). Now we have NS for PKU, congenital hypothyreoidism, congenital adrenal hyperplasia and cystic fibrosis in Slovakia.

Objective: We demonstrate actual organization algorithm of Slovak NS. Process NS begin in maternal hospital, continue in Newborn Screening Centre Laboratory (NSC SR). High positive results NSC SR are reported to Special Diagnostic Centre in the West, Middle and East of Slovakia. Middle positive results (grey zone) are reported to general practitioner, who realised second sample dry blood spot. All participations NS in Slovakia cooperate and worked with feedback.

Conclusion: Organization algorithm od Slovak NS with central laboratory is a good condition for expansion NS. We are going to pilot study for NS Rare Metabolic Disorders in 2012. 


\section{Poster session - Abstract 22}

\section{Congenital adrenal hyperplasia - clinical outcomes of neonatal screening in west Slovakia, implications for future}

\section{Pribilincová Z.}

$2^{\text {nd }}$ Dept. of Pediatrics, Comenius University Medical School, University Children's Hospital, Bratislava, Slovakia

Congenital adrenal hyperplasia due to 21 hydroxylase deficiency (CAH) is an autosomal recessive disorder of steroidogenesis. Due to its incidence and preventable potentially fatal consequences of undiagnosed $\mathrm{CAH}$ (1), there has been introduced a neonatal mass screeningby measuring 17-OHprogesterone (170HP). Difficulties might occur with interpretation of results, since steroid levels in a newborn vary according to gestational age, birth weight, general status and treatment. Neonatal screening for $\mathrm{CAH}$ has been performed in Slovakia since 2004, resulting in incidence of classical forms 1: 8000 (2). Cutoff values are adjusted to the birth weight. Beginning of the treatment shortened from average 26 days before to 10.5 days after screening. At our centre, covering 1,8 million inhabitants, there were 8 newborns diagnosed in 7 years ( 0 to 3 yearly). All were salt wasters, with genotypes in concordance with salt loosing phenotypes. Data from false positive results in years $2005-2008$ showed higher negative correlation of 17OHP levels and gestational age versus $170 \mathrm{HP}$ levels and birth weight, but with no significant difference. Age over 36th week of gestation and birth weight over $2500 \mathrm{~g}$ did not correlate with levels of $17 \mathrm{OHP}$. Out of 113 false positive newborns $68 \%$ were treated for serious postnatal complications. Our data show that screening for $\mathrm{CAH}$ needs improved assays together with clinical experience to increase accuracy of program. As shown from Middle European Society of Paediatric Endocrinology (MESPE) cooperative studies in the past, this region has good potential to elucidate the role of new methods to improve neonatal screening for $\mathrm{CAH}$.

Literature: Kovács J., Votava F., Heinze G., Sólyom J., Lebl J., Pribilincová Z. et al. Lessons from 30 Years of Clinical Diagnosis and Treatment of Congenital Adrenal Hyperplasia in Five Middle; Knapková, M., Dluholucký S.: Yearly final reports of the Neonatal Screening Centre Banská Bystrica Slovakia 


\section{Poster session - Abstract 23}

\section{MS/MS tandem for IMDs detection and screening in Slovakia}

Ostrovský I. ${ }^{1}$, Górová R. ${ }^{1}$, Addová G. ${ }^{1}$, Kubinec $R .{ }^{1}$, BlaškoJ. ${ }^{1}$, Behúlová $D .{ }^{2}$, Šaligová $J .{ }^{3}$, Halová K. ${ }^{4}$

${ }^{1}$ Dept. of Chemistry, Faculty of Natural Sciences, Comenius University, Bratislava

${ }^{2}$ Centre of Inherited metabolic diseases, Dept. of Laboratory Medicine, Dept. of Pediatrics, University Children's Hospital, Bratislava, Slovakia

${ }^{3}$ University Children's Hospital, Košice, Slovakia

${ }^{4}$ Children's Faculty Hospital, Banská Bystrica, Slovakia

At present MS tandemis worldwide used for Inherited Metabolic Disorders (IMDs) screening. MS tandem technology is still missing in the Slovak health care system. In 2009 was established Experimental Laboratory for Metabolomic Analyses (ELMA Lab, research grant ITMS 26240220007) equipped by MS tandem(TSQ Quantum QC). Since December 2010 ELMA Lab provides MS tandem analyses for IMDs observations and screening. Dry blood spot sample is collected after 72 hours of delivery and/or by purpose.In a positive case the recall is provided. At present ELMA Lab is still in an experimental mode as a specific part of existing selective screening. The capacity is about 100 samples a week. For the last 12 months about 2500 samples of newborn and/or children were investigated. Among them there were about 20 IMD positive patients diagnosed previously and monitored at present. In 2011 ELMA Lab was able to observe two new IMD positive patients (MCADD and Methylmalonicaciduria B12responsive). MS tandem show its ability and capacity to find and/or monitor IMD by properly high sensitivity and selectivity for screening and diagnose purposes. The ability to track influence of therapy and nutrition on the acylcarnitine profile was confirmed. This contributes to the quality of IMD observations and monitoring.

Acknowledgement: (ELMA Laboratory belongs to results of the research project „Výskum a vývoj nových technológii chemickej analýzy pre metabonomiku/metabolomiku“ ITMS 26240220007, granted by Operational program $R \& D$ of EU structural funds) 


\section{Poster session - Abstract 24}

Bioimpedance spectroscopy-derived body composition indices reveal lean tissue mass depletion in pediatric cystic fibrosis patients

Švekušová M., Feketeová A., Podracká L.

$1^{\text {st }}$ Dept. of Pediatrics, P. J. Šafárik University Medical school, University Children's Hospital, Košice, Slovakia

Objectives: Chronic catabolic state in malnourished CF patients leads to lean body mass depletion that is poorly detected by routine nutritional indicators. The study aimed to determine body composition changes in pediatric CF patients using bioimpedance spectroscopy (BIS) -derived indices of adipose and lean tissue mass compared to standard anthropometric evaluation.

Methods: 36 CF patients aged 5 to 18 years (mean 11.5) were enrolled in this cross-sectional study. Anthropometric measurements (height-for-age, BMI and mid upper arm circumference (MUAC)) were evaluated according to national reference data. Body composition was assessed using a BIS device (Body Composition Monitor, Fresenius). Adipose tissue index $\left(\right.$ ATI $\left(\mathrm{kg} / \mathrm{m}^{2}\right)=$ adipose tissue mass $/$ height $\left.^{2}\right)$ and lean tissue index $\left(\mathrm{LTI}\left(\mathrm{kg} / \mathrm{m}^{2}\right)=\right.$ lean tissue mass/height ${ }^{2}$ ) were used as indices of fat mass and lean mass respectively and were evaluated using manufacturer provided reference ranges for children and adolescents aged $2-18$ years. BMI, MUAC, ATI and LTI percentile $<10$ th were used as cutoff points defining nutritional compromise.

Results: Mean z scores for height, BMI and MUAC were $-0.61,-1.08$ and -1.31 respectively, with significantly lower MUAC SD in females $(-1,48$ vs. $-1,11, p<0.05)$. BMIp values $>10$ th were found in $57 \%$ males and $73 \%$ females. $17 \%$ of males and $36 \%$ of females with BMIp $>$ 10th had LTIp $<10$ th (hidden depletion of lean tissue mass). In $22 \%$ of males with BMIp $<$ 10th an isolated fat mass reduction was identified, all females with BMIp $<10$ th had lean tissue depletion. Lean tissue loss was undetectable by BMI in $22 \%$ of males and $50 \%$ of females with LTIp $<10$ th.

Conclusion: Bioimpedance spectroscopy reveals lean tissue mass depletion undetected by BMI. Hidden lean tissue loss seems to be more severe in females. 


\section{Poster session - Abstract 25}

\section{Depression, anxiety and self-esteem in relation to quality of life in patients with cystic fibrosis}

Štěpánková K. ${ }^{1}$, Nagyová I. ${ }^{2,3}$, Feketeová A. ${ }^{4}$, Takáč B. ${ }^{5}$; Kopcová L. ${ }^{6}$; Berešová E. ${ }^{7}$

${ }^{1}$ Slovak Cystic Fibrosis Association, Košice, Slovakia.

${ }^{2}$ Graduate School Košice Institute for Society and Health, Faculty of Medicine, P.J.Šafárik University, Košice, Slovakia

${ }^{3}$ Institute of Public Health - Dept. of Social Medicine, Faculty of Medicine, P. J. Šafárik

University, Košice, Slovakia

${ }^{4}$ Children's Hospital, Košice, Slovakia

${ }^{5}$ Children's Hospital, Banská Bystrica, Slovakia

${ }^{6}$ University Hospital of L. Pasteur, Košice, Slovakia

${ }^{7}$ Faculty Hospital of F. D. Roosevelt, Banská Bystrica, Slovakia

Background: Numerous studies have demonstrated that in patients with chronic disease markers of disease severity are only modestly associated with quality of life (QoL), whereas depression, anxiety and self-esteem are important correlates. Few studies of patients with cystic fibrosis (CF) have looked at the associations between these variables.

The aim of this study was to examine whether CF patients with symptoms of depression and anxiety and lower self-esteem report diminished QoL.

Methods: The sample consisted of 47 adolescents and adults ( $\geq 14$ years) with $C F$ from 4 CF

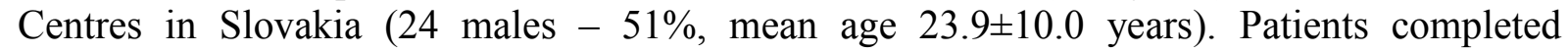
the Cystic Fibrosis Questionnaire (CFQ-R), Hospital Anxiety and Depression Scale (HADS) and Rosenberg Self-Esteem Scale (RSE). Multiple linear regression analysis was used to explore the data.

Results: After controlling for relevant sociodemographic (age, gender) and clinical variables (FEV1, BMI) symptoms of depression and anxiety were significantly associated with lowered QoL in 3 out of 12 CFQ-R domains: Emotional Functioning, Eating Disturbances and Weight. Self-esteem was found to be significantly associated with 5 CFQ-R domains: Physical Functioning, Vitality, Emotional Functioning, Social Functioning, and Health Perception; even when controlling additionally for symptoms of depression and anxiety. After entering all variables into the equation the total explained variance for CFQ-R domains varied between $9-41 \%$.

Conclusions: QoL is an important clinical outcome measure in CF. Clinicians need to be sensitive to symptoms of depression and anxiety as well as levels of self-esteem in patients with CF, because they seem to be important determinants of QoL. Psychological interventions aimed on increasing the selfesteem may potentially improve QoL among patients with CF. 


\title{
Poster session - Abstract 26
}

\section{The Slovak Huntington Association}

\author{
Kvasnicová M., Pavliková J.
}

The Slovak Huntington Association, Bratislava, Slovakia

The Slovak Huntington Association (SHA) is a non-governmental voluntary organization which associates the patients suffering from Huntington's disease (HD), their relatives and other persons who are interested in the problems of HD.

$\mathrm{HD}$ is an autosomal dominant neurodegenerative disease. The occurence of the disease is 1:10 000. Most patients with HD develop symptoms at age between 35 and 45. The average survival after the diagnosis is 16 years. The casual treatment for it does not yet exist. SHA was founded in 1994 with 22 members. At present time it has 86 members -14 affected persons, 24 persons in risk for the disease and 48 others. It is the member of International Huntington association and the member of Slovak Humanitarian aid organization.

The aims of the Association:

- To inform the patients and their families about the character of the disease, its heredity, the possibilities of genetic diagnostics, the treatment and the care for patients and family members ( consulting, medical-genetic, psychological and social care).

- To inform professional and laic public about the problems of patients with HD

- To provide the co-operation between families of patients with HD, physicians and other experts.

- To search for so far non diagnosed individuals with HD

- To create the conditions for improvement the care for patients with HD including new types of care facilities.

- To co-operate with other organizations of humanitarian character as well asa with inland and foreign partner oranizations and institutions.

The activities of the Slovak Huntington's association

- SHA organize family meetings with educational and relaxation program

- Lectures with topic on Huntington disease

- Publish the newsletter „HOPE“, articles about HD in scientific and popular journals, booklets for families with HD

SHA cooperates with clinical genetic departments in Slovakia. In spite of our effort the cooperation with neurologists, psychiatrists and practitioners is not sufficient till now. We are also not very succesful with stimulating our lay members to their own activity. Families with HD have many problems (health, social, psychological and other) According to our experiences neurologists,psychiatrists and medical practitioners have very little information and experiences with this disease.

In our country there are not special nursing units, homes, clinics or department for people with HD.

SHA try to improve the conditions of life for patients with HD and their families.

Acta Facult. Pharm. Univ. Comenianae, 2012, 59, Suppl 1 


\title{
Poster session - Abstract 27
}

\section{Diagnosis, treatment and problems of patients with Marfan syndrome}

\author{
Lukovičová A. ${ }^{1}$, Ondrejčák $M .^{1,2}$, Dušinský $R .^{1,3}$ \\ ${ }^{1}$ Slovak Marfan Association \\ ${ }^{2}$ Dept. of Clinical Genetics, District Hospital Železničná, Novapharm s.r.o Bratislava, Slovakia \\ ${ }^{3}$ Dept. of Genetics, Faculty of Natural Sciences, Comenius University, Bratislava, Slovakia
}

Diagnosis: Marfan syndrome (MFS) is a genetic disorder caused by a mutation in the gene FBN1 controlling the production of fibrillin. MFS results in weakening of connective tissue in the whole body especially in skeletal, cardiovascular and ocular systems. Dg. of MFS is complicated by broadly variable clinical manifestation. In the 2010 revised Ghent nosology, aortic root aneurysm/dissection and ectopia lentis are cardinal features. In the absence of any family history, the presence of these two cardinal features is sufficient for the unequivocal diagnosis of MFS, irrespective of the presence or absence of systemic features in other organ systems, except where these are indicative of differential diagnosis. The confirmed FBN1 mutation or combination of systemic manifestations is required when one of the cardinal features is absent.

Treatment: There is no cure for MFS. The prevention is important - heart problems: regular echocardiograms even in childhood, aorta diameter measurements, use of beta-blockers, potentially aortic replacement surgery; eyes problems: regular examinations with potential surgical solutions: cerclage, lensectomy and lens implantation. MFS includes many symptoms. Patients require treatment by qualified professionals from multiple medical disciplines.

Problems: Risk of sudden vascular events (rupture of the aorta, heart attack), vision loss, serious damage to the spine or musculoskeletal system.

Prevention: Necessary body protection by limiting of physical activity and by relaxing and regeneration at regular intervals throughout the day. Disability.

The disease is permanent with the worsening tendency; the mean age is about one third shorter. Lack of information of disease causes that MFS is often underestimated by doctors and patients.

Support: The Slovak Marfan Association (http://marfan.szm.sk) plays important role in the dissemination of information, brings together patients and relatives in Slovakia and provides the expert advice for the patients and helps to improve the medical care.

Contact: AMS, Alžbeta Lukovičová (president), Vajnorská 54, 83104 Bratislava, Slovakia. tel.: 00421 2-44 257 904,mob: 00421903 747424, marfan.sk@mail.t-com.sk 


\section{Poster session - Abstract 30}

\section{Heparin-induced thrombocytopenia}

Sokol J., Plameňová I., Chudý P., Ivanková J., Dobrotová M., Staško J., Kubisz P.

National Center of Hemostasis and Thrombosis, Jessenius Faculty of Medicine, Comenius University, Martin, Slovakia

Heparin-induced thrombocytopenia (HIT, ORPHA3325, ICD 10 D69.5) is a relatively common immune-mediated disorder with the potential for serious thromboembolic complications. It is associated with the use of unfractionated heparin (UFH) and may be defined as a decrease in platelet count during or shortly after exposure to this anticoagulant. HIT occurs in up to $5 \%$ of patients who are exposed to UFH. Characteristic signs of HIT are a drop in platelet count of $>50 \%$ during 5 to 15 days of heparin treatment and/or new thromboembolic complications during heparin therapy. HIT is caused by heparin-dependent IgG (HIT-IgG) that recognizes a complex of heparin and platelet factor 4 (PF4), leading to platelet activation via the platelet Fc gammaRIIa receptor. Regular platelet count monitoring is best suited for early diagnosis of HIT, especially if UFH is used. Functional and antigen assays are available to confirm HIT. Heparin withdrawal and treatment with an agent that directly inhibits thrombin or decreases thrombin generation should be initiated prior to laboratory confirmation because of the rapidity with which thrombotic complications occur following platelet decline. The alternative anticoagulants, danaparoid (a heparinoid), argatroban (a synthetic direct thrombin inhibitor), and lepirudin (a recombinant direct thrombin inhibitor), are available for further anticoagulation in patients affected with HIT. To reduce the risk of HIT is to use low-molecular-weight heparin (LMWH) instead of UFH, LMWH is less frequently associated with HIT.

Acknowledgement: Supported by the European Regional Development Fund (ERDF) Project CEPV II (Center of Excellence for Perinatological Research) (ITMS26220120036), Project "Support of human resources development using the most modern methods and forms of education at JFM CU in Martin"(ITMS 26110230031) and Grant Vega 1/0029/11. 


\section{Poster session - Abstract 31}

\section{Treatment of two sisters with morbus Gaucher}

Birásová $M$.

Hematologic ambulance, District Hospital Hornooravská, Trstená, Slovakia

Objective: Here are the presentation of the diagnosis, monitoring and treatment of two sisterswith type 1 Gaucher disease (GD) on hematology clinic in Trstená in 1993 - 2012.

\section{Patients:}

Case report no. 1: 16 years old patient examined by r. 1993 for light gradethrombocytopenia, and splenomegaly, exclude primary, secondary causes, of bone marrowGaucher cells, in collaboration with the Center for inherited metabolic disorders in Pragueconfirmed type 1 GD, decreased b-glucocerebrosidase, the analysis of DNA mutation $1226 \mathrm{G} / 1448 \mathrm{C}$. The progression of organomegaly and pancytopenia, especially thrombocytopenia,severe, since 1999 to enzyme replacement therapy (ERT) Cerezyme 15U/kg/2wks from 201060U/kg/2wks. The ERT improved hematologic parameters, reduction of organomegaly, decreased chitotriosidase, clinically stable.

Case report no. 2: 14 years old patient - sister of the first patient, examined in 1994 in thescreening of the family, confirmed type $1 \mathrm{GD}$, from 2004 to ERT at a dose of Cerezyme $15 \mathrm{U} / \mathrm{kg} / 2 \mathrm{wks}$, from 2010 at a dose $30 \mathrm{U} / \mathrm{kg} / 2 \mathrm{wks}$. In the treatment of withdrawal splenomegaly,laboratory adapted. Increased monitoring of pregnancy, the application of ERT is welltolerated in pregnancy, successfully completed two pregnancies in 2006, 2010.

Results: The course outlines the follow-up - initial manifestation, diagnosis and treatment of ERT, haematological, biochemical markers, the volume of the spleen, liver, skeleton,chitotriosidase. Treatment with ERT in both sisters with good effect, without complications, with the younger sister has successfully completed two pregnancies.

Conclusion: Our 20-year follow-up shows that early diagnosis and treatment of ERTsignificantly improves survival and quality of life of patients with GD. ERT can be applied in pregnancy and is well tolerated. 


\section{Poster session - Abstract 32}

\section{Tyrosinemia type I - case report}

Šebová C. ${ }^{1}$, Behúlová D. ${ }^{1}$, Fabriciová K. ${ }^{2}$, Škodová $J .{ }^{1}$, Šalingová $A .{ }^{1}$

${ }^{1}$ Centre for Inherited Metabolic Diseases, Dept. of Laboratory Medicine, Comenius University Children's Hospital, Bratislava, Slovakia

$21^{\text {st }}$ Dept. of Pediatrics, University Children's Hospital, Bratislava, Slovakia

Introduction:Tyrosinemia type $\mathrm{I}$ is an autosomal recessive disorder, early onset form presents with acute liver failure, chronic liver disease and development of rickets are characteristic for the mild form.

Case report: A 12-month-old boy presented with anemia and hepatomegaly. Abdominal ultrasound revealed multiple focal hepatic lesions suspicious for malignancy. Laboratory examinations disclosed elevated aminotransferases, hyperbilirubinemia, anemia, thrombocytopenia, impaired liver synthetic funcions (coagulopathy, hypoalbuminemia). CT scan established multiple liver focal lesions and supported unclear malignancy. However a bone marrow aspirate was normal. Markers for inherited metabolic diseases were indicated. Elevated plasma tyrosine, phenylalanine and methionine and generalised aminoaciduria were disclosed. Urinary organic acid analysis showed an increase of aromatic amino acid metabolism. A significantly elevated excretion of $\delta$-aminolevulinic acid and serum $\alpha$-fetoprotein concentration were revealed. High excretion of succinylacetone in urine was found as a diagnostic hallamark. Orfadin (NTBC) and dietary therapy was started and positive clinical and laboratory response was noticed. Tyrosinemia type I was confirmed by mutation analysis.

Conclusion: The incidence of tyrosinemia type I is $1 / 100000$ to $1 / 120000$. Reported child is only the second patient diagnosed within the last 15 years in Slovakia. Is hepatorenal tyrosinemia quite so rare in Slovakia or clinicians do not think about it? 


\section{Poster session - Abstract 33}

\section{First case of GM1-gangliosidosis in Slovakia}

Šalingová A. , Behúlová D. ${ }^{1}$, Šaligová $J .^{2}$, Petrovič $R .^{3}$

${ }^{1}$ Centre for Inherited Metabolic Diseases, University Children's Hospital, Bratislava, Slovakia

${ }^{2}$ Dept. of Pediatrics, University Children's Hospital, Košice, Slovakia

${ }^{3}$ Centre of Medical Genetics, University Hospital, Bratislava, Slovakia

GM1-gangliosidosis is a rare autosomal recessive lysosomal storage disorder caused by deficiency of acid ß-galactosidase and characterized by generalized accumulation of GM1ganglioside, oligosaccharides (OLS), keratan sulphate and their derivates in the body. Infantile GM1-gangliosidosis (type I) starts in early infancy with rapidly progressive psychomotor deterioration, facial dysmorphism, skeletal dysplasia, muscle weakness and macular cherry-red spot.

We report on a 8-month-old girl presenting profound failure to thrive, development delay, hepatosplenomegaly, hypotonia and facial dysmorphism. Diagnosis was based on high performance think layer chromatography analysis for detection of OLS in urine as well as screening spot test, quantitative determination and electrophoretic separation of glycosaminoglycans (GAG) in urine. The activity of acid ß-galactosidase was measured in blood leukocytes.

Analysis of OLS in urine showed massive excretion of fractions creating pathognomic profile for GM1-gangliosidosis. The quantitative analysis confirmed normal to moderately increased excretion of GAG (up to $76.9 \mathrm{~g} / \mathrm{mol}$ creatinine). The electrophoretic pattern discovered increased excretion of keratan sulphate. The diagnosis was proved by markedly reduced activity of acid $\beta$-galactosidase $1.4 \mathrm{nmol} / \mathrm{h} / \mathrm{mg}$ (control $80-160 \mathrm{nmol} / \mathrm{h} / \mathrm{mg}$ ). The definitive diagnosis would require also enzyme assay of neuraminidase and DNA analysis to exclude galactosialidosis or combined defect of several enzymes. 


\section{Poster session - Abstract 34}

\section{Tandem mass spectrometry- problems connected with MCADD diagnose}

Górová R. ${ }^{1}$, Addová G. ${ }^{1}$, Šaligová J. ${ }^{2}$, Behúlová D. ${ }^{3}$, Ostrovský I. ${ }^{l}$, Kubinec R. ${ }^{l}$, Blaško J. ${ }^{l}$, Chandoga $J^{4}$, Petrovič $R{ }^{4}$

${ }^{1}$ Dept. of Chemistry, Faculty of Natural Science, Comenius University, Bratislava

${ }^{2}$ University Children's Hospital, Košice, Slovakia

${ }^{3}$ Dept. of laboratory medcine, Centre of metabolic disorders, University Children's Hospital, Bratislava, Slovakia

${ }^{4}$ Institute of Medical Biology, Genetics and Clinical Genetics, Comenius University Medical School, University Hospital Bratislava, Slovakia

At present MS tandem is used for screening of severe inherited metabolic disorders. MediumChain Acyl-CoA dehydrogenase deficiency (MCADD)is disorder of mitochondrial fatty acids oxidation, with the highest prevalence in this particular group $(1: 15,000$ newborn).Early detection and diagnose of MCADD critically contributes to the declination of mortality and life quality.

In the ELMA Laboratory (research grant ITMS 26240220007)MS tandem TSQ Quantum QC in a positive mode was used.

The very first patient observed by ELMA Lab, i.e. by MS tandem laboratory in the country was children 11 month of age. The patient was hospitalized because of unconsciousness.First MS tandemanalysis detectedvery low level of acylcarnitines (ACC), without typical MCADD profile in ACC. Diagnose was supposed from organic acids profile in urine and confirmed by DNA analysis. Tandem analysis of followed DBS sample confirmed diagnose in our lab and in a screening center in abroad.Additional MS tandem analysis of newborn dried blood sample show that the is particular patient would be detected already in the newborn stage if the MS tandem was available in that moment. In another case,MS tandem show its ability to detect MCADD disorder for two twin patients of different genotypes, one homozygote and other heterozygote. Naturally, the ACC profile of heterozygote was less typical than that of homozygote. 


\title{
Poster session - Abstract 35
}

\section{Mutational spectrum in patients with suspect Wilson disease}

\author{
Lisyová J., Fischerová M., Petrovič R., Chandoga J.
}

Institute of Medical Biology, Genetics and Clinical Genetics, Comenius University Medical School, University Hospital, Bratislava, Slovakia

Wilson disease (WD) is relatively rare genetic disorder characterized by an autosomal recessive trait of inheritance and with worldwide prevalence of 1:30000. WD is caused by mutations in $A T P 7 B$ gene (13q14.3) encoding a copper-transporting ATPase type P. The function of protein is biosynthetic (delivery of copper into the secretory pathway) and homeostatic (participation in export of excessive copper from the cells). WD is characterized by progressive accumulation of copper in tissues and subsequently by toxic effect on organism. The disease affects various organs, mainly liver, brain and kidney. Important feature of this disorder is its curability (untreated disease is always fatal). Clinical symptoms of Wilson disease are very heterogenous and in relation to organ pathology there are several forms (hepatic, neurologic, psychiatric). The treatment is lifelong and application of chelate agents (D-penicilamine) and zinc therapy is recommended. The presence of broad mutational spectrum (more than 380 known mutations) gives rise to the various phenotypic manifestations and considerably complicates the diagnostics. H1069Q is the most frequent mutation in the Central Europe population. Worldwide it accounts for $60 \%$ of all noted mutations. For rapid detection of this causal mutation in the cohort of suspected patients we have used a diagnostic PCR method BI-PASA (Poláková et al., 2007). We have detected four other prevalent mutations (3402delC, 1340del4, R778G, W779X) by using PCR-RFLP (Vrábelová et al., 2005) and fragment analysis (Fischerová, unpublished). Since 2006 we have performed the molecular-genetic DNA diagnostics of Wilson disease in 523 patients and in 22 patients we have confirmed the supposed diagnosis. We have identified 17 homozygous forms (H1069Q- 14 patients, 3402delC- 1 pacient, 1340del4- 2 patients). The other patients are compound heterozygotes for H1069Q and one of the less frequent mutations (3402delC- 2 patients, 1340del4- 1 patient, W779X- 2 patients). In 54 patients we have detected only one causal mutation but the diagnosis of WD cannot be excluded because of lacking sequencing analysis (SA). In symptomatic patients with only one or none present mutation and positive biochemical and functional markers, SA is necessary for definitive diagnosing. This molecular-genetic test is offered by our Institute in presence. 


\title{
Poster session - Abstract 36
}

\section{Prader-Willi and Angelman syndroms: base and diagnostics}

\author{
Lukáčková L. ${ }^{1,2}$, Mattošová S. ${ }^{1}$, Jurkovičová M. ${ }^{1,2}$, Petrovič R. ${ }^{1}$, Chandoga J. ${ }^{1}$ \\ ${ }^{1}$ Institute of Medical Biology, Genetics and Clinical Genetics, Comenius University Medical School, \\ University Hospital Bratislava, Slovakia \\ ${ }^{2}$ Dept. of Genetics, Faculty of Natural Sciences, Comenius University, Bratislava, Slovakia
}

Prader-Willi syndrome (PWS) and Angelman syndrome (AS) are clinically distinct neurodevelopmental genetic disorders that map to 15q11-q13 locus. The primary phenotypes are attributable to loss of expression of parentaly specific imprinted genes within this region, where the gene function is dependent on parental origine. This can arise by several mechanisms. Both syndroms have incidency about 1:25000 to 1:10 000. PWS arises from the loss of function of genes exclusively expressed from the paternal allele and is characterised by severe hypotonia, hypogonadism, strabismus, scoliosis and feeding difficulties in early infancy, followed in later infancy or early childhood by excessive eating and gradual development of morbid obesity. Loss of function of genes exclusively expressed from the maternal allele causes AS characterized by severe developmental delay, microcephaly, seizures, absent or severely limited speech, gait ataxia and/or tremulousness of the limbs, and a unique behavior with a happy demeanor that includes frequent and sometimes inappropriate laughter, smiling, and excitability.

Laboratory analysis based on sodium bisulphite treatment of DNA followed by PCR using primers specific for differentially methylated sites within the SNRPN exon 1/promoter regions. Two approaches are necessary. At first, nested PCR followed by methylation sensitive restriction analysis and at second, allele specific real-time PCR with melt-curve analysis. When a diagnosis of AS or PWS is confirmed, microsatellite analysis of SNPs within the PWS/AS critical region and control markers within the rest of the 15. chromosome will often be required to distinguish between the various disease mechanisms. The biochemical access is due to hyposensitivity of GABA receptor, that leads to two or three times higher level of presynaptic $\gamma$-aminobutyric acid (GABA), that causes increase of GABA values in the serum.

Combination of these methods gives us the reliable diagnosis and it can be applied to genetic counseling and also to prenatal testing. To date, we have confirmed supposed diagnosis of PWS or AS in 18 patients from 325 tested samples.

Acknowledgment: This work was supported by grant UK/392/2010

Acta Facult. Pharm. Univ. Comenianae, 2012, 59, Suppl 1 


\section{Poster session - Abstract 37}

\section{Complex mutational analysis of the PAH gene in Slovak patients with phenylketonuria}

Polák E. ${ }^{l}$, Ficek A. ${ }^{l}$, Baldovič M. ${ }^{1}$, Feráková E. ${ }^{l}$ Šoltýsová A. ${ }^{1}$, Strnová ${ }^{2}{ }^{2}$, Ürge O. ${ }^{2}$, Kovács L. ${ }^{3}$, Kádaši L. ${ }^{l}$

${ }^{1}$ Dept. of Molecular Biology, Faculty of Natural Science, Comenius University, Bratislava, Bratislava ${ }^{2}$ Dept. of Pediatrics, Slovak Medical University, Bratislava, Slovakia

${ }^{3} 2^{\text {nd }}$ Dept. of Pediatrics, Comenius University Medical School, University Children's Hospital, Bratislava, Slovakia

This work continues previous studies to detect molecular basis of phenylketonuria in Slovak patients. DHPLC screening for all 13 exons of the PAH gene was introduced and optimized. DNA samples from 48 subjects with biochemically proven phenylketonuria were analyzed in whom direct DNA diagnostics (detection of 11 most frequent mutations) allowed to identify mutations on only one or none PKU chromosome. In this study, 16 new mutations were detected, (Q20X, F39L, R68S, F233I, R176X, P281L, R270I, T278N, R243X, A300S, I306V, W326X, L348V, S349P, E390G, Y387X), which were not known in this population as yet. Individual mutations were allocated to the appropriate phenotypic categories of phenylketonuria. The present results will be applied for routine diagnostic practice of PKU in this country. In addition to causative mutations, several genetic polymorphisms were detected in introns, but also in the exons of the PKU gene. To date, $84.4 \%$ of Slovak PKU alleles are already identified, detection of the remaining $15.6 \%$ needs additional studies and investigational approaches. 


\section{Poster session - Abstract 38}

\section{Ciliopathies - the role of primary cilia in the pathogenesis of cystic kidney diseases}

Nagyová G., Jankó V., Ilenčiková D, Kovács L.

$2^{\text {nd }}$ Dept. of Pediatrics, Comenius University Medical School, University Children's Hospital, Bratislava, Slovakia

Cystic kidney disease is one of the most frequent hereditary disorders. Autosomal dominant polycystic kidney disease (ADPKD) with the prevalence of 1:500 to 1:1000 presents usually in adulthood. The autosomal recessive form of polycystic kidney disease (ARPKD) is rarer than ADPKD but it has a severe course even in early childhood. Nephronophthisis (NPHP) is another cystic kidney disease with an autosomal dominant inheritance, which is the most common cause of end stage renal disease in patients younger than 30 years. In a comparison to ADPKD and ARPKD, in NPHP the kidney size is normal or reduced and the cysts are typically located at the corticomedullary junction. Renal histopathology reveals tubulointersticial fibrosis. NPHP might occur with retinitis pigmentosa (Senior-Løken syndrome) or hepatal fibrosis and aplasia of cerebellar vermis (Joubert syndrome) in approximately $10 \%$ of patients. Identification and a functional characteristics of altered proteins in ADPKD (polycystin-1), ARPKD (fibrocystin) and 9 altered genes in NPHP (NPHP1 to NPHP9) led to the concept of „ciliopathies“. The hypothesis suggests, that the genes, highly conserved in evolution, are expressed at the level of the cilium-centrosome complex, which when mutated, cause renal cystic disease. The primary cilia represent sensory organelles, which transmit various extracellular signals (mechanical, visual, osmotical, etc.) to regulate the cell cycle and coordinate movement of epithelial cells, polarized in the plane of the epithelial layer. In ,ciliopathies“ the dysfunction to transmit external stimuli seems to lead to abnormal cell differentiation. This theory can explain also affection of another organs and functions in NHPH, for example retinitis pigmentosa, hepatal fibrosis, ataxia, situs inversus and mental retardation. New important knowledge in relation between ciliary mediated mechanosensation and signaling pathways of cell cycle in animal models (mouse, $C$. elegans) which will help in patients care is expected in extensive research programs in this field.

Acknowledgment: This work was supported by grant VEGA 1/0995/11

Acta Facult. Pharm. Univ. Comenianae, 2012, 59, Suppl 1 


\section{Poster session - Abstract 39}

\section{The molecular diagnostics of Bartter syndrome}

Jankó $V .{ }^{l}$, Ryšavá R. ${ }^{2}$, Kovács $L .{ }^{1}$

${ }^{1} 2^{\text {nd }}$ Dept. of Pediatrics, Comenius University Medical School, University Children's Hospital, Bratislava, Slovakia

${ }^{2}$ Dept. of Nephrology, 1st Faculty of Medicine, Charles University in Prague and General University Hospital in Prague, Czech Republic

The Bartter syndrome is an inherited disease with an autosomal recessive inheritance characterised by hypokalemia, hypochloremic metabolic alkalosis, hypercalciuria and increased renin activity besides normal blood pressure values.

Case report: 3.5 years old boy with hypotrophy and growth retardation had been hospitalized with reccurent vomiting and weakness. Investigations showed metabolic alkalosis (pH 7.51) with hypokalemia $(2.28 \mathrm{mmol} / \mathrm{l})$ and hypochloremia $(88 \mathrm{mmol} / \mathrm{l})$. The plasma levels of magnesium were in physiological range $(0.96 \mathrm{mmol} / \mathrm{l})$. Urinary loss of calcium and potassium were increased (calcium/creatinine rate 1.08; fractional excretion of potassium 19.86). Patient was normotensive although the plasma renin activity was elevated $(>320$ $\mathrm{pg} / \mathrm{ml})$.

Substitution of potassium ( $3 \mathrm{mmol} / \mathrm{kg} / \mathrm{day})$ and treatment with ibuprofen $(27 \mathrm{mg} / \mathrm{kg} / \mathrm{day})$ led to stabilization of potassium serum levels $(3 \mathrm{mmol} / \mathrm{l})$ and growth improvement (boy's growth is at the 50. percentile). The genetic analysis identified mutations c. $908 \mathrm{~A}>\mathrm{C}$ and c. $1269 \mathrm{del}$ $C$ in the gene for chloride channel CLCNKB. This channel is localized in the basolateral membrane of ascendet loop of Henle.

Discussion: Hypokalemic alkalosis usually evokes the possibility of Bartter's syndrome in a patient. This hereditary disease of renal tubules is a consequence of chloride channel disorder in a thick ascendent limb of loop of Henle. There are various genetical types of Bartter syndrome, which may have different clinical symptoms, but hypokaliemic alkalosis is present in all of theese types. Manifestation of the classical form is typical in childhood and adolescence with polyuria, muscle weakness and episodes of vomiting and is caused by mutations in gene CLCNKB. Neonatal form is caused by mutations in gene NKCC2 (Type I) or ROMK (Type II), occurs in neonatal period with polyhydramnion, polyuria and episodes of dehydratation. Neonatal form in combination with deafness is a consequence of mutation in BSND gene (Typ IV) or when CLCNKB and CLCNKA genes are both affected (Typ V). Substitution of potassium and inhibition of prostaglandin synthesis decelerate the disease progression.

Conclusion: The hypokalemic alkalosis should be a signal to think about hereditary tubulopathies (Bartter or Gitelman syndrome). Early diagnosis and appropriate treatment is a presumption for regular developement of organism and enables to avoid of complications of the disease.

AcknowledgmentThis work was supported by grant VEGA 1/0995/11

Acta Facult. Pharm. Univ. Comenianae, 2012, 59, Suppl 1 


\section{Poster session - Abstract 40}

\section{Hereditary pancreatitis in children - molecular genetic testing}

Töröková K., Čierna I., Kovács L.

$2^{\text {nd }}$ Dept. of Pediatrics, Comenius University Medical School, University Children's Hospital, Bratislava, Slovakia

Hereditary pancreatitis is a very rare disease with early onset, slow progress and variable clinical presentation. Inheritance is autosomal dominant with incomplete, approximately 80 percent penetrance and variable expressivity. Mutation of the PRSS1 gene (cationic trypsinogen gene) increases the autocathalytic conversion of trypsinogen to active trypsin that increases the autodestruction of the pancreas leading to chronic inflammatory changes. Other genes associated with chronic pancreatitis are PRSS2 (anionic trypsinogen gene), SPINK1 (serin protease inhibitor - Kazal type 1) and CFTR (cystic fibrosis transmembrane conductance regulator).

Our aim is to point out the therapeutical pitfalls and possibilities of genetic diagnosis with case studies of three patients with chronic pancreatitis. The onset of the disease was at ages 8, 14 and 16 with strong abdominal pain from acute exacerbation of until that time asymptomatic chronic pancreatitis. Chronic inflammatory changes led to pancreatic fibrosis, pseudocysts and calcifications in the parenchyma and along the pancreatic duct. All of them required surgery according to fulminant course of the acute exacerbation. DNA analysis was indicated to confirm the inherited form of chronic pancreatitis with autosomal dominant inheritance from mutations in genes PRSS1 and SPINK1. One of the patients had mutation of PRSS1 gene $-\mathrm{R} 122 \mathrm{H}$ and in two patients mutation of SPINK1 gene - N34S in heterozygous form was revealed.

These case studies draw attention to the importance of diagnostic of inherited forms of chronic pancreatitis. After exclusion of other causes included cystic fibrosis, the indication for PRSS1 and SPINK1 mutation testing in symptomatic patients should be one of the following criteria: a) recurrent unexplained attacks of acute pancreatitis and a positive family history (in two of our patients acute pancreatitis appeared in a first-degree family members in young adulthood), b) unexplained chronic pancreatitis and a positive family history, c) unexplained chronic pancreatitis without a positive family history after exclusion of other causes, d) unexplained pancreatitis episode in children. Patients with confirmed hereditary pancreatitis are at risk for developing exocrine and endocrine insufficiency and are at 50-fold increased risk of pancreatic cancer; therefore the prevention of the complications is possible with early diagnostic, follow-up and education of these children. 


\section{Poster session - Abstract 41}

\section{Constitutional mismatch repair-deficiency syndrome and high-grade brain tumors in siblings with biallelic MSH6 mutations}

Ilenčíková D. ${ }^{1}$, Sejnová $D .^{2}$, Jindrová ${ }^{2}{ }^{2}$, Babál $P .^{3}$

${ }^{1} 2^{\text {nd }}$ Dept. of Pediatrics, Comenius University Medical School, University Children's Hospital, Bratislava, Slovakia

${ }^{2}$ Dept. of Pediatric Oncology, Comenius University Medical School, University Children's Hospital, Bratislava, Slovakia

${ }^{3}$ Dept. of Pathology, Faculty of Medicine, Comenius University, Bratislava, Slovakia

Constitutional mismatch repair-deficiency syndrome (CMMR-D) is a rare autosomal recessive disease, first reported in 1999. It is characterized by hematological malignancies, brain tumors and tumors of the large intestine with an early formation. Skin maculae with diffuse margins and irregular pigmentation are similar to café au lait present in neurofibromatosis 1, and they may occur together with hypopigmented areas. Development of this syndrome is caused by biallelic mutations in genes that are associated with Lynch syndrome (hereditary non-polyposis colorectal cancer - HNPCC). MLH1, MSH2, MSH6 and PMS2 genes belong to the mismatch repair system and play a basic role in the genome integrity maintenance by correction of single base pair mismatches after DNA replication. In the heterozygous state, the mutation of one of these genes causes HNPCC. Biallelic mutations of the aforementioned genes are characterized by occurrence of the first malignancies (brain tumors, leukemias) at the age of 2 and occurrence of other types of tumors with increasing age. Wimmer and Kratz summarized the contribution of mutations in $M L H 1 / M S H 2$ and MSH6/PMS2 to the development and the spectrum of malignant tumors. The authors used data from 52 reports covering 92 CMMR-D patients and they performed genotype-phenotype correlation output from the last 12 years. We present a case with CMMR-D caused by novel homozygous MSH6 mutations leading to gliomatosis cerebri and T-ALL in an 11-year-old female and glioblastoma multiforme in her 10-year-old brother, both with rapid progression of the diseases. A literature review on brain tumors in CMMR-D families shows that they are treatment-resistant and lead to early death. Identification of patients with CMMR-D is critical, and specific cancer screening programs with early surgery are recommended. 


\section{Poster session - Abstract 42}

\section{Arterial tortuosity syndrome - a rare cause of severe pulmonary hypertension}

\section{Zemánková J., Venczelová Z., Hrebík M., Mašura J.}

The National Institute of Cardiovascular diseases, Cardiovascular children's centre Bratislava, Slovakia

Introduction. Arterial tortuosity syndrome is an extremely rare autosomal recessive connective tissue disorder caused by mutation in SLC2A10 gene, localised on chromosome 20q13. It is characterized by elongation and tortuosity of the large and medium-sized arteries, propensity to aneurysm formation, vascular dissection, potentially resulting in multiple vascular stenoses. Associated clinical features consist of excessively stretchable skin, herniae, skeletal abnormalities, joint laxity and dysmorphic features. Less than 80 cases have been described.

Case presentation. We report on 2 patients (siblings, with consanguineous parents) with suspicious arterial tortuosity syndrome; the genetic testing for this syndrome is unavailable in our country. Both children have a craniofacial dysmorphism and presented with severe pulmonary arterial hypertension, exercise-induced dyspnea and cyanosis.

Our first patient was a 6 year old girl $(20 \mathrm{~kg})$, with severe pulmonary hypertension on echocardiography and during cardiac catheterization and with numerous peripheral pulmonary artery stenoses; she was non-responder to inhaled nitric oxide. We also performed an interventional procedure with balloon dilation of 2 stenotic vessels of the right pulmonary artery using standard and cutting balloons. There was a partial relief of vascular stenoses, another interventional procedure with stent implantation is scheduled.

Our second patient is the brother of the previous patient, 4 year old $(13,8 \mathrm{~kg})$; on echocardiography and on angiography, the findings are very similar to the findings of his sister, with suprasystemic pulmonary artery pressures and multiple peripheral pulmonary artery stenoses.

Both children are treated with disease-specific therapy of pulmonary hypertension (endothelin-receptor antagonist - Bosentan), which is a long-term treatment.

Conclusion. When treating patients with severe pulmonary arterial hypertension, in differential diagnosis, we should consider the arterial tortuosity syndrome as a potential very rare cause. 


\section{Poster session - Abstract 43}

\section{Severe combined immunodeficiency}

Čižnár P. ${ }^{1}$, Horáková $J .^{2}$, ŠufliarskaS. ${ }^{2}$, Bod'ová $I^{2}$, Švec $P{ }^{2}$, Veghová $L .^{2}$

${ }^{1} 1^{\text {st }}$ Dept. of Pediatrics, Comenius University Medical School, University Children's Hospital, Bratislava, Slovakia

${ }^{2}$ Bone marrow transplantation unit, Comenius University Medical School, University Children's Hospital, Bratislava, Slovakia

Severe combined immunodeficienciy (SCID) is a heterogeneous group of genetic diseases in the development or function of T cells and variably in B cells, which in the first year of life cause severe or life threatening infectious complications. Without therapeutic intervention the disease ends up fatally within the first year of life nearly in all cases. During the time of regular nonselective BCG vaccination in Slovakia, all patients developed symptoms of disseminated infection by this vaccination strain. With ending this practice in Slovakia from 1.1.2012 prognosis could improve, if an early diagnosis is established. Due to rare prevalence of SCID (1:50000) a high rate of clinical suspicion is necessary. Though not sensitive enough a simple screening methods are a detailed family history of early death in infancy for infectious complication or an unexpected lymphopenia in the blood count, failure to thrive and lower respiratory infection. In some states of USA a screening testing for SCID has been introduced using a RT-PCR method.

In the University Children's Hospital in Bratislava there were in the last 8 years diagnosed and treated 6 children with SCID. In three cases adenosine deaminase deficiency was detected by enzyme analysis, in one case common gamma chain deficiency (X-SCID) was present, in other boy a T-B-NK + defect was detected with negative RAG1, RAG2 and Artemis gene defect analysis and in one case a more delayed phenotype of a $\mathrm{T}-\mathrm{B}+\mathrm{NK}+$ deficiency was confirmed in a 2 year old girl. Four children have been successfully transplanted, using allogenic hematopoietic stem cells (2 HLA identical, 2 matched unrelated donors). Mean time of hospital stay was 7 months, the longest one nearly 16 months. SCID is a medical emergency in pediatrics. From the time of the first suspicion the child needs specific approach, prophylactic conservative therapy and rapid transportation to the specialized centre. The causal therapy is considered to be hematopoietic stem cell transplantation, gene therapy and in some forms replacement therapy. Treatment in the first quarter of the first year, before the more profound complications develop, has a favorable prognosis. 


\section{Poster session - Abstract 44}

\section{Rare skin diseases}

Danilla T.

Dept. of Dermatoveneorology, Centre for Treatment of Epidermolysis bullosa, Comenius University Medical School, University Children's Hospital, Bratislava, Slovakia

This group of rare diseases is characterised by: a) Incidence of less than 5 to 10000 inhabitants, b) Life-threatening or chronically debilitating and c) Genetically determined This group of diseases in dermatovenereology has several representants.

The most common and most serious diseases are genetically determined causing failure of cohesion of the skin - epidermolysis.

Individual forms have their own genetic nature of pathological - anatomical substrate, different clinical manifestations, course, treatment and prognosis.

Epidermolysis bullosa junctionalis (EBJ) is the most serious form of which Herlitz and EBJ with pyloric stenosis often lead to death from complications within a few years after birth. Another severe form of Epidermolysis bullosa dystrophica (EBD) is Hallopeau-Siemens, which also affects the lining of internal organs and leads to complications. Despite good medical care it leads to invalidization at a young age (loss of fingers and toes, narrowing of the digestive tract, urogenital tract, superinfection, sepsis, nutritional disorders, and movement). Less severe forms are non Herlitz EBJ, EBD dominanta - which heal with atrophy of the skin. The least severe forms belong to Epidermolysis bullosa simplex (EBS). The incidence is unknown, it is rare disease. In Slovakia 15 children with EBD are registered.This group of genodermatoses causes not only serious clinical manifestations, but also social problems and interferes with visible skin changes in to psychosocial relationships, and significantly reduces the patients quality of life and that of his family as well.

Children suffering from rare diseases need special medical care involving expensive diagnosis, long time treatment, prevention and especially treatment of complications. They require a specific approach to create better conditions for their life.

Medical cooperation between many specialists is necessary, because these diseases affect not only the skin but the whole body. Cooperation with the pediatrician and the dietetist is needed to treat the complications and to impaire nutrition, due to damage of the digestive tract. Also cooperation with the surgeon and urologist is needed in cases of strictures, or with the plastic surgeons in remodeling the fingers, the rehabilitation physician and psychologist to ensure proper development.

The Department of Pediatric dermatovenereology elaborates documents for the purpose of the correct approach for the diagnosis and treatment of epidermolysis bullosa. After the approval of the Ministry of Health it was issued in a special edition of the Bulletin of the Ministry of Health in Slovakia, 31.12 th. 2009 as Professional guideline on the diagnosis and treatment of epidermolysis bullosa.

Poster shows schemes of disturbed microstructure, damaged loci of genes and clinical picture of epidermolysis. 


\section{Poster session - Abstract 46}

\section{Multiple occurrence of MCAD deficiency - case report of a family}

Šaligová J.', PotočñákováL. ${ }^{1}$, Behúlová D. ${ }^{2}$, Chandoga $J .^{3}$, Bzdúch $V .^{2}$, Andrejková M. ${ }^{1}$, Maníková M. ${ }^{1}$

${ }^{1}$ University Children's Hospital, Košice, Slovakia

${ }^{2}$ University Children's Hospital, Bratislava, Slovakia

${ }^{3}$ Centre of Medical Genetics, Faculty Hospital, Bratislava, Slovakia

Medium - chain acyl - CoA dehydrogenase (MCAD) deficiency is the most common fatty acid oxidation disorder. Its frequency has been estimated at one in 6000 to 10000 Caucasians. More than $90 \%$ of patients are homozygous for the most prevalent mutation - A $985 \mathrm{G}$.

Affected individuals appear to be normal until Reye-like episode, often rapidly progressive metabolic crisis which is provoked by a period of $8-12-16$ hours of fasting during intercurrent illness, after surgery or just for a delayed breakfast. MCAD deficiency can be also a reason of SIDS (sudden infant death syndrome). Classical laboratory presentation is hypoketotic hypoglycemia, typical profile of acylcarnitines (elevated hexanoylcarnitine and mainly octanoylcarnitine) and organic acids (dicarboxylic aciduria with glycine conjugates).

Authors present a case report of a family with multiple MCAD deficiency. Non consanguineous parents of Gipsy origin had 11 children. 5 cases of MCAD deficiency were present in the family. The diagnose was proven in 2 children post mortem according the typical acylcarnitine and organic acid profile. Both children died during few hours after acceptance in a hospital with the clinical presentation of sever hypoglycaemia with progressive hepatopathy and metabolic decompensation resistant to the therapy. One child suddenly died at home during respiratory illness in the age of 7 months. The diagnose was proven in 2 children by DNA testing. They were homozygotes for mutation A 985 G. 3 children are heterozygotes, 3 children are healthy homozygotes. Authors pointed ou the problems with diagnostics and compliance in certain families as it was also in described case. Fatty acid oxidation defect was suspected in a boy in his almost 3 years of age after a Rey -like episode, but the family did not undergo the investigations. Finally we persuaded them to the testing only after the death of third child. Second day after the first ambulatory visit one of the affected children needed hospitalization because of the developing of metabolic crisis. Fortunately she was saved. At the time of diagnosis both living children have the signs of metabolic decompensation with serious carnitine depletion. After carnitine supplementation and changing of dietary habits their metabolic compensation is quite satisfactory except intercurrent illnesses, quite often hospitalization was necessary. 


\title{
Poster session - Abstract 47
}

\section{Needs of People with Neuromuscular Disorders in the field of Care and Equipment Provision}

\author{
Jankyová S. ${ }^{l}$, Korčeková M. ${ }^{1}$, Duračinská M. ${ }^{2}$, Foltánová $T .{ }^{l}$ \\ ${ }^{1}$ Dept. of Pharmacology and Toxicology, Faculty of Pharmacy, Comenius University, Bratislava, \\ Slovakia \\ ${ }^{2}$ Organization of Muscular Dystrophy in the Slovak Republic, Bratislava, Slovakia
}

Introduction:Muscular dystrophy (MD, ICD 10 G71) refers to a group of more than 30 genetic neuromuscular diseases that cause progressive weakness and degeneration of skeletal muscles. Generally the symptoms involve muscle weakness and loss of muscle tissue. The prevalence varies about $0.3-0.5$ in 10000 people in the EU. In the Slovak republic no data exists, however the National Centre for Health Information (NCHI) states, that in the year 2010 there were about 9014 patients with myoneural junction diseases G 70 - G73 4111 (male) and 4903 (female).The data about the mortality are even cruder, in the year 2010, 429 patients died due to the neural dystrophies. Similar situation is in diagnostics as well as care for patients with rare neuromuscular diseases. Since information is missing, we prepared a short perspective of available medical devices for patients with neuromuscular diseases.

Methods: The list of medical devices was prepared according the pricing list provided by the Ministry of Health, with the cooperation with the patient's organisation. The medical devices were divided in two groups, medical devices for children and adults. Every group of the medical devices was divided into subgroups according their compensation ability (hygiene, positioning, transport, respiratory devices). Orthotics as well as invasive pulmonary ventilation were not included, because these are hard to quantify.

Results: There are generally 255 medical devices for adults, which are approved, and could be suitable for patients with neuromuscular diseases, out of them $35 \%$ are fully reimbursed by public health insurance. In children the situation is similar 243 medical devices and $32 \%$ fully reimbursed. According the law, in every subgroup must be at least one fully reimbursed alternative, however this must be not applicable in rare neuromuscular diseases. The average patients costs are $28 \%$ [ $4-81 \%]$. In children the average patient costs are $29 \%$ [9-57\%]. The more expensive medical devices have higher reimbursement, however the criteria are to general and don't take into account the prognosis of the disease (power wheelchair vs. manual one) as well as the fact, that they have compensate the inborn disease and enable the patients equal and the most possible integration into the society.

Conclusion: The wilderness of medical devices for patients with neuromuscular diseases seems to be rich, on the other hand to general to be oriented and sufficient for rare prevalent and highly individualised diseases. Existence of National Program for Rare Diseases as well as Centre of Expertise would be of big advantage to increase the accessibility of appropriate and individual medical care, including medical devices.

Literature: The Diagnosis and Management of Duchenne Muscular Dystrophy, A guide for families, TREAT - NMD Neuromuscular Network, Standards of Care for Spinal Muscular Atrophy, TREAT NMD Neuromuscular Network, Consensus Statement for Standard of care in Sinal Muscular Atrophy, $J$ of Child Neurol 2007:22 (p. 1027 - 1049), Kočová, H; Kopecká, D: Přehled kompenzačnich pomucek u tělesného a kombinovaného postižení v dětském věku, 2011

Acta Facult. Pharm. Univ. Comenianae, 2012, 59, Suppl 1 


\section{Poster session - Abstract 48}

\section{Molecular genetic analysis of NPHS2 gene in children with steroid resistant nephrotic syndrome}

Jankó V. ${ }^{1}$, Habrmanová M. ${ }^{2}$, Baldovič $M .^{2}$, Kadási $L .{ }^{2}$, Kovács $L .{ }^{2}$

${ }^{1}$ 2nd Dept. Pediatrics, Comenius University Medical School, University Children's Hospital, Bratislava, Slovakia

${ }^{2}$ Dept. of Molecular Biology, Faculty of Natural Sciences, Comenius University, Bratislava, Slovakia

The annual incidence of idiopathic nephrotic syndrome (NS) in children in Europe has been estimated to be $1-3$ per 100,000 children, with a cumulative prevalence of 16 per 100.000 children. About $90 \%$ of patients are steroid responsive, with a favorable long-term prognosis. The remaining $10 \%$ of children who do not respond to corticosteroids are particularly at risk for extrarenal complications of NS and the development of end-stage kidney disease (ESKD), which occurs in 30-40\% of children with steroid-resistant NS (SRNS) after a follow-up of 10 years. It has been suggested that inherited structural defects of the glomerular filtration barrier are responsible of up to $20 \%$ of SRNS cases, thereby emphasizing the crucial role of the podocyte in the pathogenesis of glomerulopathies. Indeed, mutations in genes highly expressed in podocytes have been found in nephrin, podocin and CD2AP, which are the main structural elements of the slit diaphragm and which are encoded by NPHS1, NPHS2 and $C D 2 A P$ gene, respectively.

Patients and results: A total of 25 children aged 4 to 17 years with sporadic nephrotic syndrome resistant to 6 weeks steroid treatment $\left(60 \mathrm{mg} / \mathrm{m}^{2}\right)$ were screened for podocin mutations. One of them presented homozygous mutation that was associated with early onset of proteinuria and variable renal lesions, including one case with mesangial $\mathrm{C} 3$ deposition. Single mutations of podocin were found in two steroid-resistant cases. All three patients had the same mutation (V108M) and all responded to cyclosporin.

Conclusion: The pathogenetic implication of single podocin defects per se in proteinuria must be further investigated in view of the possibility that detection of a second mutation could have been missed. A suggested alternative is the involvement of other gene(s) or factor(s). Molecular diagnostics of genetic defects has an important therapeutic implication due to ineffectively of immunosuppressive treatment in these cases. Treatment with cyclosporin A or rituximab may be advised thanks to a direct effect of these agents on the podocyte cytoskeleton. 


\section{Poster session - Abstract 49}

\section{Enzyme replacement therapy of Fabry disease in Slovakia}

Kákošová $V^{1}$, Hlavatá $A .^{2}$, Bzdúch $V^{3}$

${ }^{1}$ Dept. of Pharmacy, University Children's Hospital, Bratislava, Slovakia

${ }^{2} 2^{\text {nd }}$ Dept. of Pediatrics, Comenius University Medical School, University Children's Hospital, Bratislava, Slovakia

${ }^{3} 1^{\text {st }}$ Dept. of Pediatrics, University Children's Hospital, Bratislava, Slovakia

Fabry disease (FD) is an X-linked, hereditary metabolic disorder characterized by a defect in the degradation of glycosphingolipids (defect of lysosomal enzyme, $\alpha$-galactosidase A). Fabry disease belongs to rare disorders, so-called ,orphan diseases“. The reported prevalence varies between 1 and 5 in 100000 , but the true prevalence may be underestimated. FD takes a progressive course and the prognosis, if untreated, is bleak. Clinical symptoms, starting with acute and chronic pain, neurological symptoms of the extremities, usually arise in childhood (4 - 10 yrs). Diarrhea, nausea, vomiting, hypohidrosis, skin lesions and corneal changes are common. With increasing age, cardiac and cerebrovascular abnormalities, together with a gradual deterioration of renal function, are very frequent.

A major breakthrough came in 1989, when the genetic code of $\alpha$-galactosidase was deciphered, enabling the enzyme to be produced laboratory. Two commercial products of $\alpha-$ galactosidase A are available for ERT. Both are produced artificially from genetically engineered cells: cultured human fibroblasts in case of agasidase- $\alpha$ (REPLAGAL ${ }^{\mathrm{TM}}$ ); and Chinese hamster ovary cells in the case of agalsidase- $\beta$ (FABRAZYME ${ }^{\circledR}$ ) but their long term safety and efficacy are still being investigated.

All patients with FD can be involved in the Fabry Registry - a global, observational, and voluntary program designed to collect clinical data related to the onset, progression, and treated course of the disease. All patients are eligible to participate in the Registry, regardless of whether they are receiving ERT and irrespective of the commercial product with which they are being treated.

Slovak patients with FD are treated in Centre for Inherited Metabolic Diseases at the Children's University Hospital in Bratislava. First two male patients started with recombinant human $\alpha$-galactosidase therapy in year 2003. They were involved in multicenter, open-label study of low dose maintenance treatment of Fabrazyme. The first, at that time 53year-old patient, refused further therapy after end of the study (2005), the other, then 26 years old, continues in the ERT. There are at present another two patients with FD; a 30-year-old man is treated with agalsidase- $\beta$, and a 62 -year-old woman with agalsidase- $\alpha$.

Clinical stabilisation was achieved in all the treated patients. Safety of the ERT is continuously monitored, no serious adverse events have occurred yet. FD was also confirmed in two male children without any clinical manifestations and therefore no ERT has been used. 


\section{Poster session - Abstract 50}

\section{Methylmalonic aciduria with signs of Asperger syndrome}

Šaligová J. ${ }^{1}$, Potočňáková L., Rosenbergerová T., Strnová J., Behúlová D. ${ }^{2}$, Mrázová L. ${ }^{5}$, Geržényiová M. ${ }^{6}$, Zeman $J^{5}$

${ }^{1}$ University Children's Hospital, Košice, Slovakia

${ }^{2}$ University Children's Hospital, Bratislava, Slovakia

${ }^{3}$ Slovak Medical University, Bratislava, Slovakia

${ }^{4}$ District Hospital Bardejov, Slovakia

${ }^{5}$ Dept. of hereditary metabolic disorders, District Hospital Prague, Czech Republic

Background: Methylmalonic aciduria (MMA) is pathogenetically and clinically heterogeneous group of disorders of intermediary metabolism. Asperger syndrome is a disorder of social interactions associated with limited stereotypical and repeated spectrum of interests and activities.

Case report:: Authors present a 20 years old patient with MMA due to a mutation in apoenzyme of methylmalonyl-CoA-mutase. The disease manifested at the age of 4 months with typical signs of organic acidurias and the patient started to keep the standard therapy. First years of his life are characterised by metabolic decompensations during infections. Pronounced regression of attacks of metabolic derangement is characterised for second decade of life, with a normal physical and neurological development. Psychopathologic symptomatology became predominating - transitory depression, unequal performance in verbal and non - verbal abilities, ADHD syndrome with specific learning disability especially in mathematics, emotional immaturity and signs of Asperger syndrome. Patient has serious problems with social interactions, special - even limited spare-time activity and interest in trains and buses, he spends hours by travelling alone and knows traffic schedules by heart. Actually his laboratory results correspond with a compensated state of MMA.

Conclusion: Autism belongs to clinical symptoms of MMA, therefore we suppose Asperger syndrome belonging to pervasive disorders is a rare consequence of MMA found in our patient. 


\title{
Poster session - Abstract 51
}

\section{Medical devices in treatment of epidermolysis bullosa in the Slovak republic How expensive they really are?}

\author{
Korčeková M. ${ }^{1}$, Ramljaková B. ${ }^{2}$, Pšenková $M .^{3}$, Foltánová $T .^{1}$ \\ ${ }^{1}$ Dept. of Pharmacology and Toxicology, FacultyofPharmacy, ComeniusUniversity, Bratislava, \\ Slovakia \\ ${ }^{2}$ Debra Dystrophic epidermolysis bullosa research association, Bratislava, Slovakia \\ ${ }^{3}$ Pharm-In, spol. s r.o., Bratislava, Slovakia
}

Epidermolysisbullosa (according ICD 10 Q81.9) is a hereditary (autosomal dominant/recessive), rare disease which is linked with mutations in gene for collagen, type VII - COL7A1 gene. The main symptoms are fragile, highly sensitive skin in different areas of the body, depending on the type of EB.

The prevalence is extra low, ranges from $0.01-0.09$ in 10000 people in European Union. There is no precise data about prevalence of EB in the Slovak republic, and these data are not specially followed by the National Centre for Health information (NCHI).

Although several drugs are under research, there is no sufficient treatment for EB. The serious course of the disease requires regular use of medical devices. Indeed the rarity of the disease, patients with EB face several problems in the Slovak health care system, which can be characterized by extemporaneousness.

Aim:To bring more light into the situation the aim of this work is to summarize all medical devices, which patients require for monthly treatment and to adjust their costs from the point of view of the health insurance as well as patients.

Methods: The list of medical devices needed for monthly treatment of patient with two types of EB (EBJ - junctional epidermolysis bullosa and RDEB - recessive dystrophic epidermolysis bullosa) was provided by the Slovak patients' organization DEBRA. The prices of all medical devices were from the official pricing list provided by the Ministry of Health. These were compared with minimum wage in the Slovak republic in the year 2012. Other statistics comes from NCHI.

Results: We created a clear table of medical devices, with all the administrative codes and monthly demands, which could simplify the work to all, who are involved in the treatment of EB. There are approximately 23 different medical devices, mainly dressing material, topical antiseptics and basic nutrition which patients need in monthly care. The monthly costs for patient with RDEB are 365 euro, whereas the costs for EBJ are approximately 568 euro. Patients' costs represent $44 \%$ of minimum wage in the Slovak republic and $35 \%$ of the hospitalization reimbursements (depending on the department and Health insurance company). In the patients with EBJ minimum wage doesn't even last to cover monthly cost of necessary medical devices (1.2 times higher than minimum wage) however they form approximately $33 \%$ of the hospitalization reimbursements.

Conclusion:There is a big discussion about the financing in the Slovak national health care at all, and this is even more urgent in rare diseases. Thanks to the VAT reduction (10\%) total price of medical devices was reduced across, however the enormous demand make them 
financially devastating for patients and their families as well as cost ineffective for the health care system.

\section{Poster session - Abstract 52}

Molecular diagnostics based on cDNA sequencing of NF1 gene andgenotype-fenotype correlation in optic nerve gliomas in Slovak NF1 patients

Rybárová A. ${ }^{1}$, Némethová M. ${ }^{2}$, Hlinková K. ${ }^{3}$, Hlavatá A. ${ }^{1}$, Kovács L. ${ }^{1}$, Kádaši L. ${ }^{4,2}$, Zat'ková A. ${ }^{4,2}$, Ilenčíková D. ${ }^{l}$

${ }^{1} 2^{\text {nd }}$ Dept. of Pediatrics, Comenius University Medical School, University Children's Hospital, Bratislava, Slovakia

${ }^{2}$ Dept. of Molecular Biology, Faculty of Natural Sciences, Comenius University, Bratislava, Slovakia

${ }^{3}$ Dept. of Cancer Genetics, National Cancer Institute, Bratislava, Slovakia

${ }^{4}$ Laboratory of Genetics, Institute of Molecular Physiology and Genetics, Bratislava, Slovakia.

Introduction: Neurofibromatosis type 1 (NF1; OMIM 162200) is one of the most commonautosomal dominant genetic disorders (incidence 1: 3000). Optic nerve gliomas (ONG) arepresent in about $15 \%$ patients with NF1. We focused on finding genotypefenotypecorrelations in a cohort of Slovak patients with NF1 and optic glioma who are at higher risk ofdeveloping a severe form of neurofibromatosis type 1

Materials and methods: We evaluated 52 patients meeting the diagnostic criteria for NF1and divided them into two groups 1.) with ONG (21 patients). 2. without ONG (30 patients).All of them underwent a clinical examination focused on NF1 and molecular diagnostics ofNF1 gene using standard protocol based on RNA (Messiaen, L. \& Wimmer, K. Karger, Basel, 2008)

Results: All patients with ONG and without ONG had café-au-lait spots. In comparisonbetween these two groups, the incidence of freckling $(95 \% / 28,5 \%)$, brain hamartomas $(71 \% / 23,3 \%)$ neurofibromas $(70 \% / 50 \%)$ Lisch nodules $(30 \% / 20 \%)$ was significantly higher inthe group with ONG compared to the group without ONG. A half of mutations found in theONG group were located in the range of first 5'tertile - first 16 exones. 15 of them were notpreviously described. In the group without optic gliomas only $27 \%$ were distributed in thefirst 5 'tertile of the NF1 gene.

Discussion: Our data confirm clustering of mutations in the 5' tertile of NF1 gene in patientswith optic nerve glioma sugguested by Sharif et al in the work published in the Journal ofMedical genetics in 2011. Patients with optic nerve gliomas have higher incidence of otherNF1 features. The diagnostic method employed proved very effective with $80 \%$ of successful mutations detections.

Acknowledgment: Grant support VEGA: Komplexná mutačná analýza génu zodpovedného za neurofibromatózu typ 1, 2010-2012, 2/0104/10 


\section{Poster session - Abstract 53}

\section{Variable clinical picture of Wilson disease}

Šaligová J. ${ }^{1}$, Potočňáková L. ${ }^{1}$, Majorová E. ${ }^{1}$, Ackermanová K. ${ }^{2}$, Drahovská I. ${ }^{2}$, Vasilová A. ${ }^{3}$, Genčík A. ${ }^{4}$, Andrejková $M .^{1}$

${ }^{1}$ University Children's Hospital, Košice, Slovakia

${ }^{2}$ University Hospital, Košice, Slovakia

${ }^{3}$ Gendiagnostika s.r.o., Slovakia

${ }^{4}$ Medgene s.r.o, Slovakia

Background: Wilson disease is an autosomal recessive disorder in which copper accumulates in tissues due to deficit of hepatic ATPase. Clinical picture is extremely variable. Hepatic manifestation is the most common in childhood. Neuropsychiatric disorder appears in adulthood.

Case report: We present a case of a family with variable clinical picture in 2 siblings. 14 years old boy was sent with 3 years history of unexplained hepatopathy first revealed during acute gastritis. Detailed examinations were performed including ceruloplasmin, which was normal during first year. Accidently 17 years old brother was present at a first visit. $\mathrm{He}$ presented with hypertonic-hypokinetic syndrome which developed during 3 months and was explained by psychiatric treatment for anxiety and depression with progressive cognitive deterioration and rigidity. Suspected Wilson disease in both was confirmed by patognomic laboratory findings (low ceruloplasmin, high urine copper excretion), MRI findings in older one and definitely by DNA analysis. Because of persistent hyperbilirubinemia and otherwise negative findings in their sister all the siblings were examined and Gilbert syndrome was confirmed.

Conclusion: Clinical and laboratory variability during course of the disease and even in the same genotype may cause confusion in diagnostics. Therefore repeated laboratory investigation is strongly recommended. 